\title{
OPEN State dependent effects on the frequency response of prestin's real and imaginary components of nonlinear capacitance
}

\author{
Joseph Santos-Sacchi ${ }^{1,2,4 凶}$, Dhasakumar Navaratnam ${ }^{1,2,3} \&$ Winston J. T. Tan ${ }^{1}$
}

The outer hair cell (OHC) membrane harbors a voltage-dependent protein, prestin (SLC26a5), in high density, whose charge movement is evidenced as a nonlinear capacitance (NLC). NLC is bell-shaped, with its peak occurring at a voltage, $V_{h}$, where sensor charge is equally distributed across the plasma membrane. Thus, $V_{h}$ provides information on the conformational state of prestin. $V_{h}$ is sensitive to membrane tension, shifting to positive voltage as tension increases and is the basis for considering prestin piezoelectric (PZE). NLC can be deconstructed into real and imaginary components that report on charge movements in phase or 90 degrees out of phase with $A C$ voltage. Here we show in membrane macro-patches of the $\mathrm{OHC}$ that there is a partial trade-off in the magnitude of real and imaginary components as interrogation frequency increases, as predicted by a recent PZE model (Rabbitt in Proc Natl Acad Sci USA 17:21880-21888, 2020). However, we find similar behavior in a simple 2-state voltage-dependent kinetic model of prestin that lacks piezoelectric coupling. At a particular frequency, $F_{i s,}$ the complex component magnitudes intersect. Using this metric, $F_{i s,}$ which depends on the frequency response of each complex component, we find that initial $V_{h}$ influences $F_{i s}$ thus, by categorizing patches into groups of different $V_{h}$ (above and below $-30 \mathrm{mV}$ ) we find that $F_{i s}$ is lower for the negative $V_{h}$ group. We also find that the effect of membrane tension on complex $\mathrm{NLC}$ is dependent, but differentially so, on initial $\mathrm{V}_{\mathrm{h}}$. Whereas the negative group exhibits shifts to higher frequencies for increasing tension, the opposite occurs for the positive group. Despite complex component trade-offs, the low-pass roll-off in absolute magnitude of NLC, which varies little with our perturbations and is indicative of diminishing total charge movement, poses a challenge for a role of voltage-driven prestin in cochlear amplification at very high frequencies.

Prestin (SLC26a5) is a membrane-bound voltage-dependent protein ${ }^{1,2}$, whose activity drives outer hair cell $(\mathrm{OHC})$ electromotility $(\mathrm{eM})^{3}$, the latter yet considered to provide for cochlear amplification at very high acoustic frequencies (50-100 kHz). Evidence supporting high frequency electromotility stems from whole-cell microchamber experiments and mechanical measures in vivo during electrical stimulation within the organ of Corti ${ }^{4-6}$. Nevertheless, recent observations against this concept have been made, indicating lower pass behavior ${ }^{7-11}$. Regardless, the voltage dependent activity of prestin itself has been well characterized over the years ${ }^{12,13}$, typically analyzed by evaluating membrane capacitance $(\delta \mathrm{Q} / \delta \mathrm{V})$ (i.e., NonLinearCapacitance, NLC). The proteins' aggregate sensor charge displays a sigmoidal Boltzmann $(\mathrm{Q} / \mathrm{V})$ relationship, being quantitatively characterized by $\mathrm{V}_{\mathrm{h}}$, the voltage at half maximal charge redistribution across the membrane (near $-40 \mathrm{mV}$ ); $z$, the apparent unitary charge (near 0.75 ); and $\mathrm{Q}_{\max }$ the maximum charge of a given protein population within the membrane. The parameter $z$ is alternatively viewed as a voltage sensitivity, providing a slope factor of the Q/V function (near $35 \mathrm{mV}$ ), which indicates a very shallow voltage dependence. Thus, the full range of prestin's voltage

${ }^{1}$ Surgery (Otolaryngology), Yale University School of Medicine, BML 224, 333 Cedar Street, New Haven, CT 06510, USA. ${ }^{2}$ Neuroscience, Yale University School of Medicine, 333 Cedar Street, New Haven, CT 06510, USA. ${ }^{3}$ Neurology, Yale University School of Medicine, 333 Cedar Street, New Haven, CT 06510, USA. ${ }^{4}$ Cellular and Molecular Physiology, Yale University School of Medicine, 333 Cedar Street, New Haven, CT 06510, USA. ${ }^{\square}$ email: joseph.santos-sacchi@yale.edu 
responsiveness spans over $300 \mathrm{mV}$. Of course, prestin is not unique in generating NLC, as channels whose voltage sensors produce displacement currents show similar behavior ${ }^{14}$.

Prestin has been deemed a piezoelectric (PZE) protein since Iwasa discovered tension sensitivity of NLC under whole cell (WC) voltage clamp ${ }^{15}$. Indeed, prestin's piezoelectric coefficient is extraordinary, estimated to be four orders of magnitude greater than man made elements ${ }^{16}$. Under WC voltage clamp, $\mathrm{V}_{\mathrm{h}}$ shifts rightward and peak NLC decreases as membrane tension increases ${ }^{15,17,18}$. In membrane patches, however, peak NLC (or $\mathrm{Q}_{\max }$ ) does not alter despite substantial shifts in $\mathrm{V}_{\mathrm{h}}{ }^{7,19}$. The likely reason why apparent $\mathrm{Q}_{\max }$ is decreased in WC mode is because tension delivery is inhomogeneous ${ }^{19}$, and differentially impinges on multiple independent micro domains of prestin ${ }^{20}$. The disparate summation in WC measures of NLC therefore shows changes in apparent $z$ resulting in a reduced peak NLC. Again, the reduction in total charge with tension is not a characteristic of prestin itself, as membrane patch studies clearly show.

Recently, Rabbitt ${ }^{21}$ suggested that the PZE nature of prestin provides power to the cochlear amplifier that correlates with the imaginary component of NLC, that component of charge movement that alters phase in response to viscous load. Such modelled behavior of prestin sensor charge movement, which for a piezo component is coupled to imposed stress, was predicted to account for the apparent incongruity of prestin's low-pass roll-off in the absolute magnitude of complex $\mathrm{NLC}^{7}$ and the expected high frequency behavior of prestin required by cochlear modelers to account for high frequency cochlear amplification. Here we thoroughly evaluate the real and imaginary components of NLC in OHC macro-patches and find partially reciprocal (not of equal magnitude) trade-offs in those components across frequency that coincide with Rabbitt's model results. We do not use the word reciprocal to intimate any reciprocity between electrical and mechanical interactions, since we made no simultaneous mechanical measures during our patch recordings, notwithstanding the general consensus that displacement currents, hence NLC, signify movements of charged residues in voltage dependent proteins ${ }^{22}$. However, we did previously explore interactions between electrical and mechanical behavior in whole $\mathrm{OHCs}^{8}$. Despite the similarity between the PZE model's complex NLC behavior and our data, we also find that a simple 2-state kinetic model lacking piezoelectric coupling provides similar trade-offs in complex components across frequency. In our biophysical data, we also observed significant effects of initial state, characterized by initial $V_{h}$, on the frequency response of the reciprocal trade-offs. Furthermore, membrane tension differentially alters that frequency response depending on initial state. Whether such observations are in line with PZE theory remain to be determined.

\section{Results}

Rabbitt ${ }^{21}$ modeled prestin and its NLC as a piezoelectric process based on first principles. NLC can be deconstructed into real and imaginary components ${ }^{23}$, since it is defined as the frequency-dependent membrane admittance $\left(Y_{m}^{*}(\omega)\right)$ divided by $i \omega$, where $\omega=2 \pi f$ and $i=\sqrt{-1}$,

$$
C_{m}^{*}(\omega)=\frac{Y_{m}^{*}(\omega)}{i \omega}=\frac{B_{m}^{*}(\omega)}{\omega}-i \frac{G_{m}^{*}(\omega)}{\omega},
$$

providing a real capacitive component and an imaginary conductive component. On removing stray capacitance (by subtraction, see "Methods" section) and linear capacitance (by Boltzmann fitting, see "Methods" section) from $C_{m}^{*}(\omega)$, prestin-associated complex $\operatorname{NLC}(\omega)$ is obtained.

With his PZE model, Rabbitt predicted that the imaginary component of complex NLC should increase over frequency, as the real component decreases. We previously reported on the real and imaginary components of complex $\mathrm{NLC}^{7}$ and concluded that the imaginary component was small and essentially frequency independent. However, we did not fit both complex components to Boltzmann functions to unambiguously extract details of those components. In that paper, we focused our attention on the absolute magnitude of complex NLC, i.e., $\sqrt{\operatorname{Re}(N L C)^{2}+\operatorname{Im}(N L C)^{2}}$, which is comparable to all previous measures of OHC/prestin NLC.

Here we explore in detail both components in 25 macro-patches of the OHC lateral membrane, first under zero pipette pressure, namely in the absence of externally applied membrane tension. For the 3D plots, black dots depict average data, and magenta dots depict standard error (+SE). The multicolored shading is provided by the interp surface plot function in Matlab, and the red and blue solid lines depict Boltzmann fits (see "Methods" section) at selected frequencies.

Figure 1 shows that indeed, Rabbitt's modelling prediction concerning our data that he references is borne out. There appears to be a trade-off between real (Fig. 1A) and imaginary (Fig. 1B) component peak magnitude across frequency, especially revealed in the extracted 2-state fits of NLC (Fig. 1C). Superimposed lines in green and magenta are the PZE model responses at $V_{h}$ from Rabbitt ${ }^{21}$, which shows general agreement within this bandwidth. Figure 1D shows the absolute magnitude of NLC that continuously decreases across frequency. Thus, while there are reciprocal trade-offs between the real and imaginary NLC components, the trade-off is not fully reciprocal; that is, the overall kinetics of prestin (governed by a host of molecular loads) slows.

Figure 2 (top panel) highlights the relationship between real and imaginary components of NLC. Here we plot extracted $\mathrm{Q}_{\max }$ from fitted NLC components across frequency. The trade-off in component magnitude shows that at a particular frequency $\left(\mathrm{F}_{\mathrm{is}}\right)$, the two intersect, and above that frequency the imaginary component will dominate. Rabbit ${ }^{21}$ modeled that the imaginary component will eventually reduce to zero after peaking, but here (because of our limited frequency interrogation range) we use $F_{\text {is }}$ to explore influences on NLC frequency response (see below). Figure 2 (middle panel) also shows that the voltage sensitivity $(z)$ of the two components decreases in parallel across frequency, possibly indicating an increasingly impeded sensor charge movement within the membrane plane. In other words, voltage-induced conformational switching of prestin is depressed as frequency increases. 
A

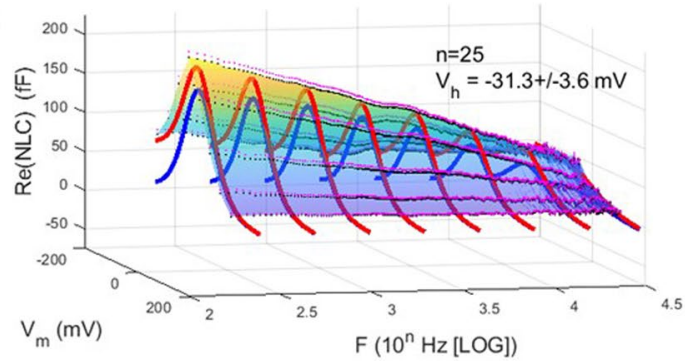

C

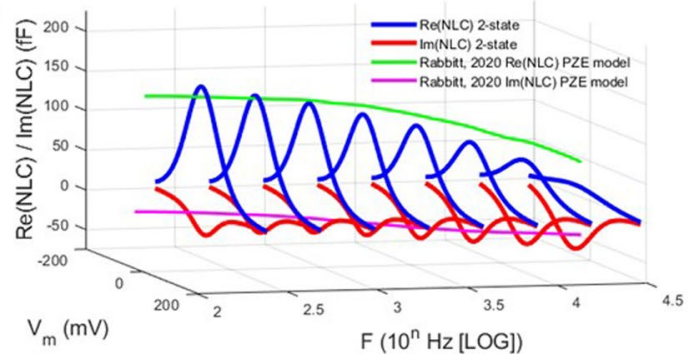

B

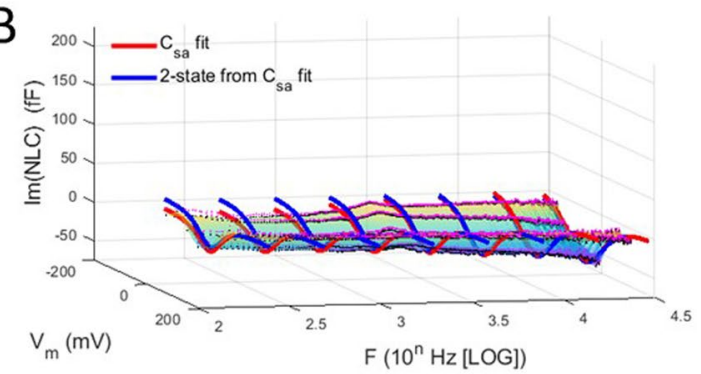

D

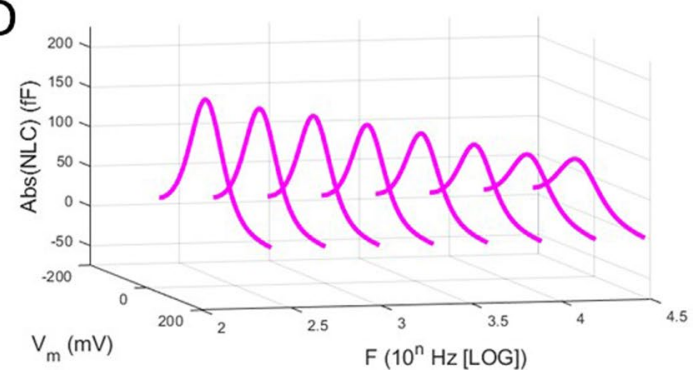

Figure 1. Real and imaginary components of patch NLC. (A) Real component of complex NLC across interrogating frequency. Note continuous decrease as frequency increases. Fits are made to Eq. (1) to extract the 2-state (blue line) response at selected frequencies. $\mathrm{V}_{\mathrm{h}}$ is average between 0.244 and $2.44 \mathrm{kHz}$. (B) Imaginary component of complex NLC. In this case, magnitude increases with frequency, but there is not a full reciprocal trade-off with the real component. (C) Plot of the 2-state fits across frequency. Superimposed is the PZE model response at $\mathrm{V}_{\mathrm{h}}$ of Rabbitt (2020), which shows general agreement within this bandwidth. (D) Plot of the absolute magnitude of NLC, namely, $\sqrt{\operatorname{Re}(N L C)^{2}+\operatorname{Im}(N L C)^{2}}$, indicating a continuous reduction of prestin activity across frequency.

Another interesting observation that this fitting exercise exposes relates to the offset $\left(\Delta C_{\mathrm{sa}}\right)$ in capacitance at negative voltages, which is uniformly observed in all previous studies reporting on OHC NLC. Indeed, when we first observed this offset and developed the method used to fit the response ${ }^{24}$, we thought it was solely due to surface area alterations as prestin changed states from expanded to contracted. Surface area change in prestin embedded membrane was first reported by Kalinec et al. ${ }^{25}$. However, the magnitude of $\Delta \mathrm{C}_{\mathrm{sa}}$ is greater than that expected for surface area changes alone in prestin. Thus, it likely includes changes to overall dielectric properties of the membrane, as well. Figure 2 (bottom panel) compares the offsets in real and imaginary component fits by plotting the difference between the two. The magnitude of $\Delta \mathrm{C}_{\mathrm{sa}}$ is little affected across frequency, and, in fact, it can be seen in Fig. 1A,B that the offset is virtually absent in the imaginary component of NLC. Thus, the charge movement associated with the offset is solely capacitive in nature.

Do these real and imaginary component trade-off observations necessarily indicate that prestin is piezoelectric? Figure 3 shows that the same basic behavior observed in our data is recapitulated by a simple 2-state kinetic model with voltage-dependence only, i.e., both real and imaginary NLC components are observed, with trade-offs in magnitude across frequency.

This simple 2-state model for charged prestin particles possesses an expanded (X) and compact (C) state, with the population of prestin molecules redistributing during changes in membrane voltage.

$$
\mathrm{X} \underset{\beta}{\stackrel{\alpha}{\rightleftharpoons}} \mathrm{C} \text {. }
$$

The forward and backward rates ( $\alpha, \beta$, respectively) are governed solely by changes in membrane voltage $\left(V_{m}\right)$ about a characteristic potential, $V_{h}$, where particles (charges) are distributed equally on either side of the membrane field. $z$ denotes the voltage sensitivity or unitary particle charge ( $\mathrm{e}^{-} \mathrm{X}$ distance travelled perpendicular to the membrane field). F, R and T have their usual meanings.

$$
\begin{aligned}
& \alpha=\alpha_{0} \exp \left(\frac{z F\left(V_{m}-V_{h}\right)}{2 R T}\right), \\
& \beta=\beta_{0} \exp \left(\frac{z F\left(V_{m}-V_{h}\right)}{2 R T}\right) .
\end{aligned}
$$

In Fig. 3A we show results from simulations with both rate constants $\left(\alpha_{0}=\beta_{0}\right)$ equal to $45,000 / \mathrm{s}$, and in Fig. 3B equal to $90,000 / \mathrm{s}$. $\mathrm{V}_{\mathrm{h}}$ was set to $-40 \mathrm{mV}$. These rate constants provide characteristic time constants $\left(\operatorname{tau}=1 /\left(\alpha_{0}\right.\right.$ $\left.+\beta_{0}\right)$ ) for charge movement in the time domain at the potential $V_{h}$. In the frequency domain, the magnitude response is a single Lorentzian (equivalent to a first-order RC response, whose transfer function displays the 

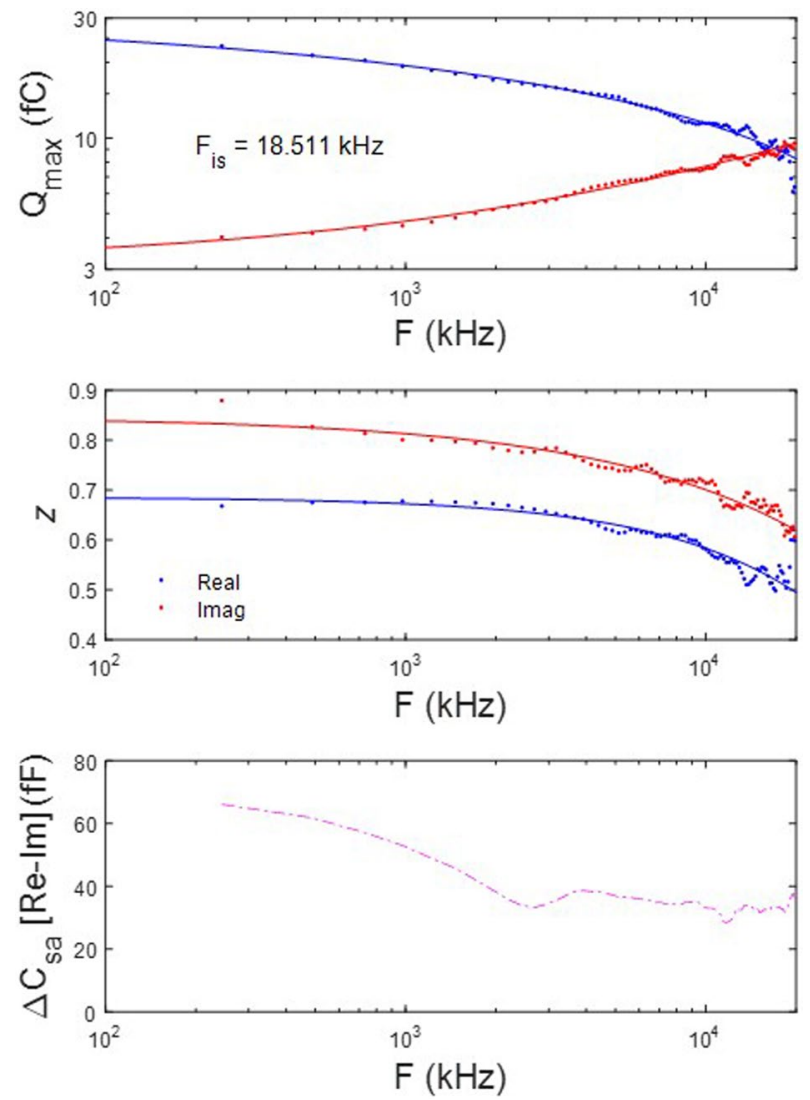

Figure 2. Frequency dependence of complex NLC components. Top panel Plot of fitted $\mathrm{Q}_{\max }$ of 2-state components of complex NLC. The real and imaginary component magnitudes intersect at a particular frequency, $\mathrm{F}_{\text {is. }}$. Here the imaginary component absolute magnitude is plotted. Fits (solid lines) are with Eq. (2). Red is real component; blue is imaginary component. $F_{\text {is }}$ is $18.5 \mathrm{kHz}$. Middle panel Plot of the Boltzmann parameter, $z$. Both decrease in parallel with frequency, with the imaginary component being smaller, indicating a shallower voltage sensitivity. Bottom panel Plot of difference between real and imaginary $\Delta \mathrm{C}_{\mathrm{sa}}$, showing some low frequency dependence. Figure $1 \mathrm{~A}, \mathrm{~B}$ indicate that $\Delta \mathrm{C}_{\mathrm{sa}}$ is largely absent in the imaginary component.

same complex behavior); the cut-off frequency at $V_{h}$ is defined as $F_{c}=1 /(\operatorname{tau} \times 2 \pi)$, where the response falls to 0.707 of the initial low frequency magnitude (or correspondingly where a phase lag of 45 degrees develops). In Fig. 3A,B, in addition to the complex component plots, the absolute magnitude of NLC at $\mathrm{V}_{\mathrm{h}}$ is shown with red lines, and the red circles mark the measured cut-off frequency, which agrees with that calculated from the utilized rate constants. The real component of complex NLC is lower pass than the absolute value. Thus, it is the absolute value of NLC that appropriately characterizes the model's frequency response, and also that of the biophysical data. We have previously used the absolute magnitude of complex NLC to characterize prestin frequency response in macro-patches.

Additionally, in Fig. 3 we illustrate the validity of our method of subtraction of a nonlinear DC conductance from our patch admittance data prior to subsequent analysis. With a nonlinear DC conductance included in the model (Fig. 3C), like that we find in membrane patches, the imaginary component at low frequencies is distorted. Following removal (Fig. 3D), complex NLC is equivalent to the model without such conductance (Fig. 3A). In the 2-state model, overall NLC behavior arises due to the exponential voltage-dependent transition rates between prestin's expanded and contracted states. Altering the rate constants will shift the cut-off and the component magnitudes (compare Fig. 3A,B), like alterations of the time constant for an RC response. There is no intrinsic piezoelectric coupling between voltage and tension embodied in this simplest 2-state model, nor in a first order RC circuit. But, without the delays introduced in those conformational transitions (i.e., in a simple ultra-fast 2-state model), no imaginary component exists within our interrogation bandwidth. Thus, the requirement of piezoelectricity in prestin is not firmly established by the trade-off in real and imaginary components of NLC that we find across stimulus frequency.

The data we have presented thus far are from averaged NLC, but we know that $\mathrm{NLC}_{\mathrm{h}}$ can vary among cells (and patches) due to a variety of reasons, e.g., membrane tension ${ }^{15,17,18}$, anions ${ }^{26-28}$, temperature ${ }^{29-31}$, initial voltage conditions $^{32,33}$, and membrane cholesterol content ${ }^{34,35}$. Indeed, prestin likely presents characteristics that vary along the length of the cell ${ }^{20}$, where functional expression (e.g., $\mathrm{V}_{\mathrm{h}}$ ) can be non-homogenous ${ }^{36,37}$. Consequently, we sought to characterize the dependence of NLC real and imaginary component behavior on prestin's initial 
A
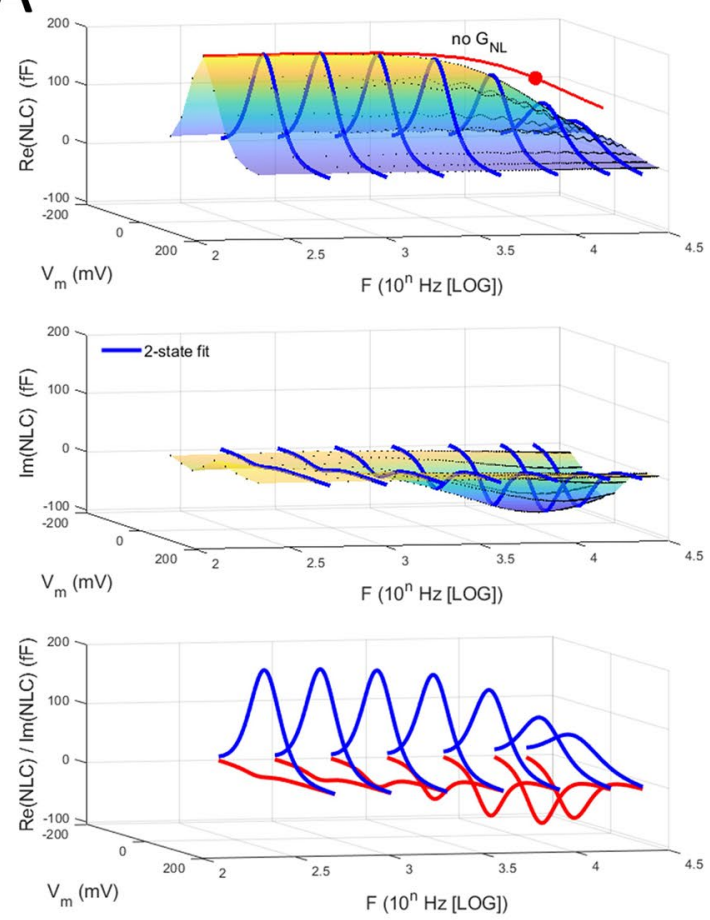

C
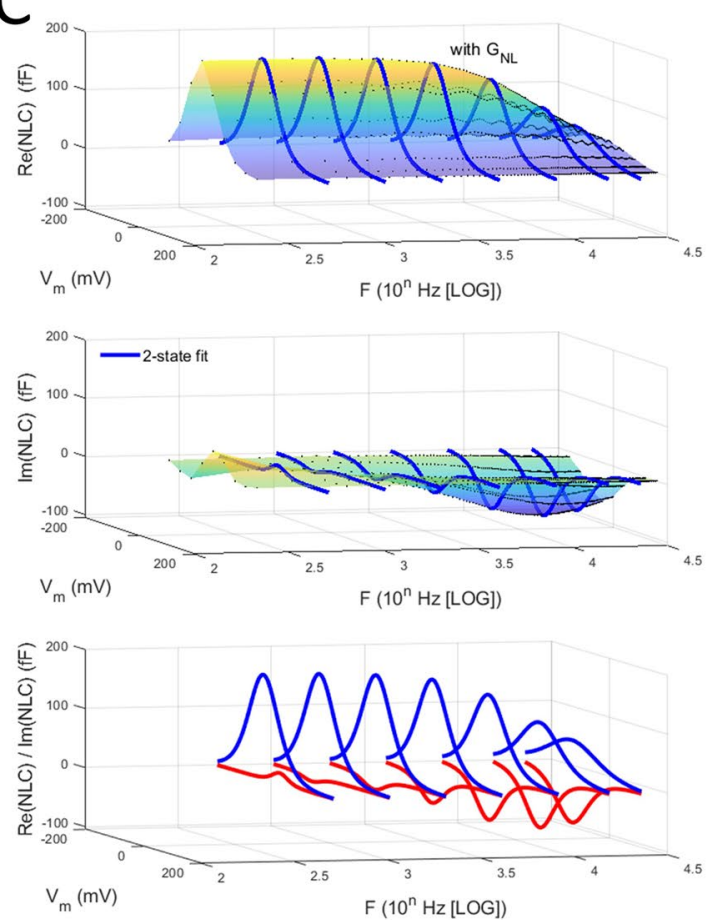

B
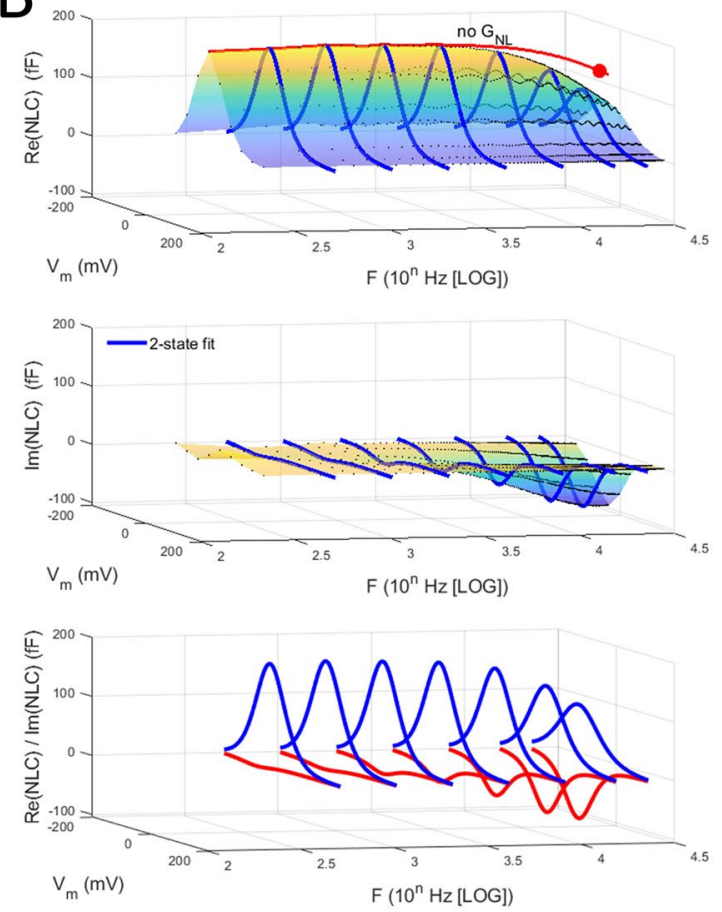

$\mathrm{D}$
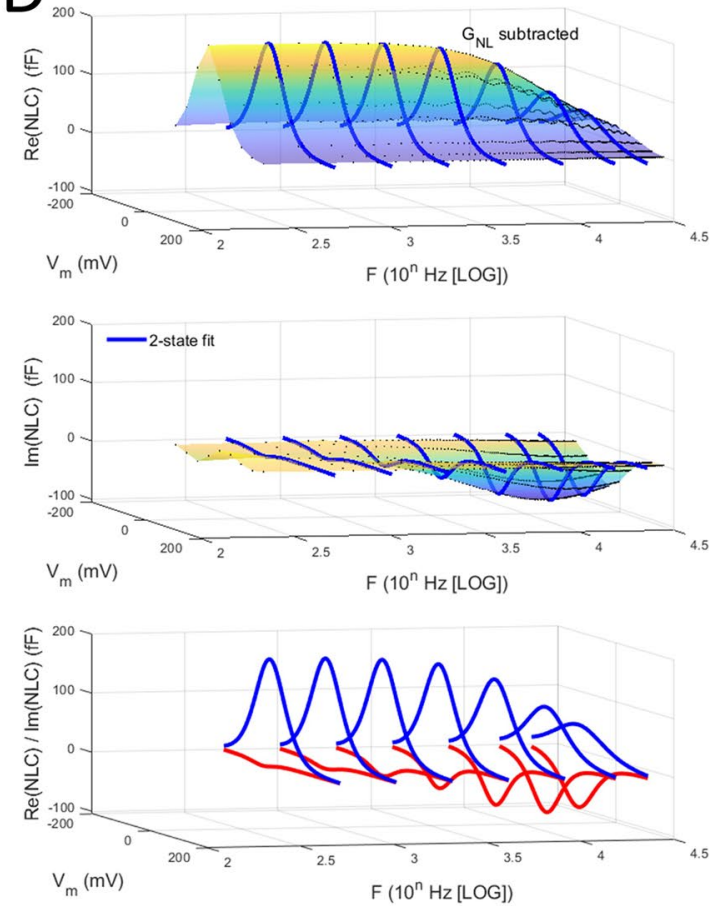

Figure 3. Real and imaginary components of NLC generated by a solely voltage-dependent 2-state kinetic model (see "Results" section for details), with characteristic cut-offs at $V_{h}$ dependent on transition rates, $\alpha$ and $\beta$. (A) simulations with both rate constants $\left(\alpha_{0}=\beta_{0}\right)$ equal to $45,000 / \mathrm{s}$, and (B) with both rate constants equal to $90,000 / \mathrm{s}$. In addition to the complex component plots, the absolute magnitude of NLC at $\mathrm{V}_{\mathrm{h}}$ is shown with red lines. The rates provide characteristic time constants $\left(\operatorname{tau}=1 /\left(\alpha_{0}+\beta_{0}\right) ; 11.1 \mu\right.$ s and $5.6 \mu$ s, respectively) for charge movement in the time domain at the potential $V_{h}$. In the frequency domain, the cut-off frequency at $V_{h}$ is defined as $F_{c}=1 /(\operatorname{tau} \times 2 \pi), 14.3$ and $28.6 \mathrm{kHz}$, respectively. The filled red circles mark the cut-off frequencies. (C) Same model as in (A), but with an additional nonlinear DC conductance (designed to be like that in our patch data) which distorts the low frequency response of the imaginary component. (D) After removing the DC conductance with the approach detailed in the "Methods" section, the response is essentially the same as in (A), where no DC conductance is included, confirming the validity of our approach to remove any residual DC conductance in our patch data. 
A

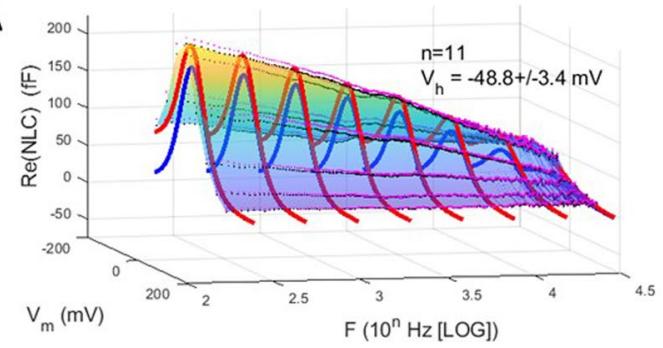

C

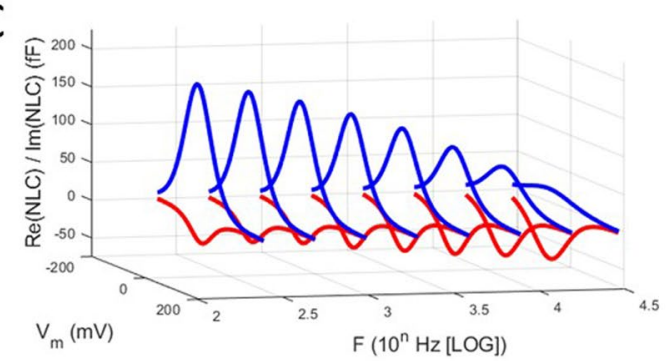

B

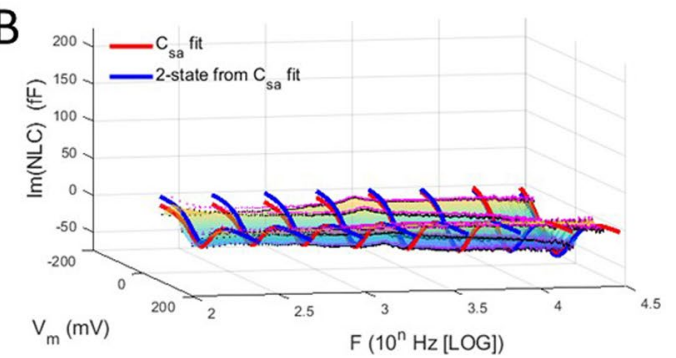

$\mathrm{D}$

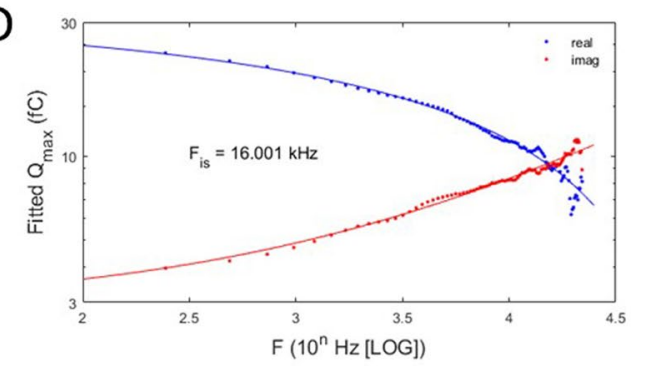

Figure 4. Complex NLC patch data for patches categorized with $\mathrm{V}_{\mathrm{h}}$ more negative than $-30 \mathrm{mV}$. (A-C) Legends to Fig. 1A-C analogously apply here. (D) Plot of fitted $\mathrm{Q}_{\max }$ of 2-state components of complex NLC. The real and imaginary component magnitudes intersect at a particular frequency, $\mathrm{F}_{\mathrm{is}}$. Here the imaginary component absolute magnitude is plotted. Fits (solid lines) are with Eq. (2). Red is real component; blue is imaginary component. $\mathrm{F}_{\text {is }}$ is $16 \mathrm{kHz}$.

A

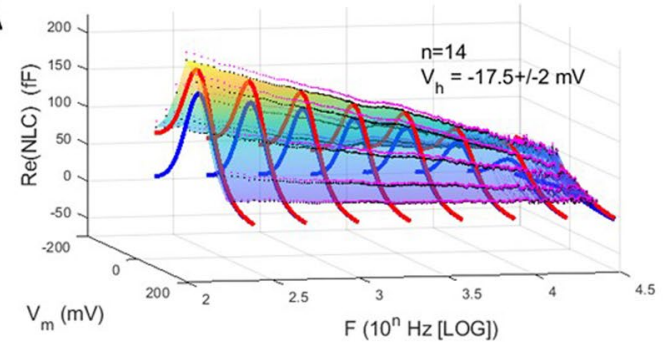

C

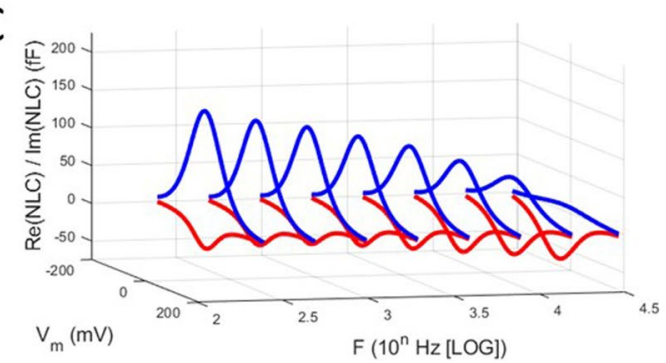

B

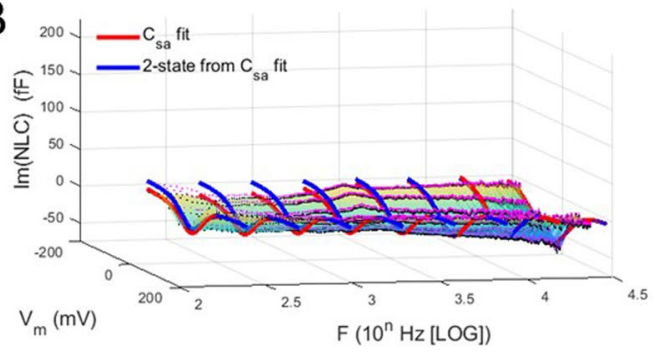

D

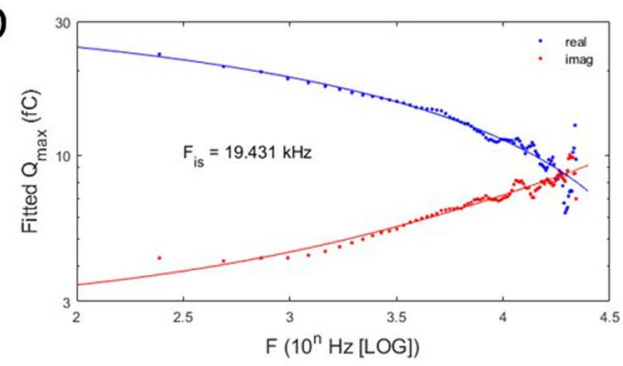

Figure 5. Complex NLC patch data for patches categorized with $\mathrm{V}_{\mathrm{h}}$ more positive than $-30 \mathrm{mV}$. (A-C) Again, legends to Fig. 1A-C analogously apply here. (D) Plot of fitted $\mathrm{Q}_{\max }$ of 2-state components of complex NLC. The real and imaginary component magnitudes intersect at a particular frequency, $\mathrm{F}_{\mathrm{is}}$. Here the imaginary component absolute magnitude is plotted. Fits (solid lines) are with Eq. (2). Red is real component; blue is imaginary component. $\mathrm{F}_{\text {is }}$ is $19.4 \mathrm{kHz}$.

state; namely, as typified by initial $\mathrm{V}_{\mathrm{h}}$, which reports on the distribution of proteins in either the expanded or contracted states. First, we categorized NLC data into groups possessing $\mathrm{V}_{\mathrm{h}}$ values above and below $-30 \mathrm{mV}$.

Figures 4 and 5 show these categorized averaged responses. Means of $\mathrm{V}_{\mathrm{h}}$ for the two groups are $-48.8 \pm 3.4 \mathrm{mV}$ $(\mathrm{n}=11)$ for the negative range group, and $-17.5 \pm 2 \mathrm{mV}(\mathrm{n}=14)$ for the positive range group. Differences between the frequency response of the two groups are apparent, especially in imaginary components (Figs. 4A-C, 5A-C). These differences are highlighted by plotting the intersection of the fitted $\mathrm{Q}_{\max }$ magnitudes of each NLC component (Figs. $4 \mathrm{C}, 5 \mathrm{C}$ ), where the intersection frequency $\left(\mathrm{F}_{\mathrm{is}}\right)$ is at a lower frequency for the negative range group $(16 \mathrm{kHz})$ than the positive range group $(19.4 \mathrm{kHz})$. Figure 6 recategorizes $\mathrm{V}_{\mathrm{h}}$ into 3 groups, and clearly shows a nonlinear increase of the intersection frequency with more depolarized initial $\mathrm{V}_{\mathrm{h}}$ conditions. 


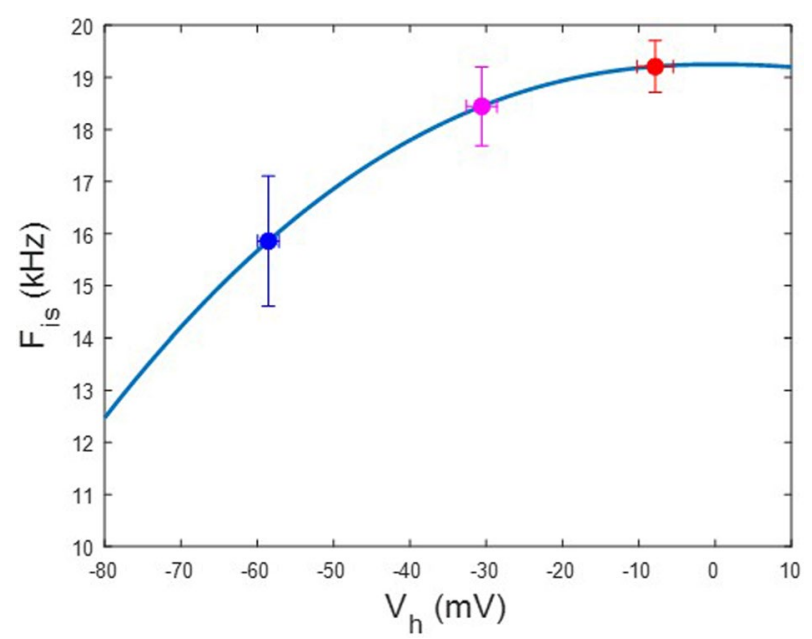

Figure 6. $\mathrm{F}_{\text {is }}$ for complex NLC patch data for patches recategorized into $3 \mathrm{~V}_{\mathrm{h}}$ groups. Error bars are SE. Note that as $V_{h}$ is more negative, $F_{i s}$ increases nonlinearly. This shows that $V_{h}$ influences the frequency responses of real and imaginary components of complex NLC.

Membrane tension is well known to shift $\mathrm{V}_{\mathrm{h}}$ of OHC NLC. To investigate the influence of tension-induced $\mathrm{V}_{\mathrm{h}}$ shift on real and imaginary components of NLC, we altered the tension delivered to the macro-patch membrane (Fig. 7). In 7 patches we were able to incrementally alter pipette pressure (thus, membrane tension) from 0 to $-2,-4,-6,-8$, and $-10 \mathrm{~mm} \mathrm{Hg}\left(0\right.$ to $-1.22 \mathrm{kPa}$ ). Though substantial shifts in average $\mathrm{V}_{\mathrm{h}}$ occur (from $-42.8 \pm 8$ to $-9.1 \pm 11.8 \mathrm{mV}$ ), only slight changes in the real or imaginary components of complex NLC are readily visible. However, upon plotting intersecting frequencies (lower panels), clear changes in $\mathrm{F}_{\text {is }}$ are obvious. Increasing tension causes a shift in $\mathrm{F}_{\text {is }}$ to higher frequencies. For $0 \mathrm{mmHg}, \mathrm{F}_{\text {is }}$ is $12.9 \mathrm{kHz}$, whereas $-10 \mathrm{mmHg}$ pressure shifts $\mathrm{F}_{\text {is }}$ to $28.3 \mathrm{kHz}$. Does initial $\mathrm{V}_{\mathrm{h}}$ factor into these responses?

As with our $V_{h}$ categorization observations made above, we sought to determine the influence of initial $V_{h}$ on tension effects. To this end, we categorized the patches into groups with initial $\mathrm{V}_{\mathrm{h}}$ above and below $-30 \mathrm{mV}$. First, Fig. 8 illustrates that tension susceptibility depends on initial $\mathrm{V}_{\mathrm{h}}$, with the positive group (red symbols; $4.28 \mathrm{mV} / \mathrm{mmHg}$ ) showing $\mathrm{V}_{\mathrm{h}}$ shift sensitivity nearly twice that of the negative group (blue symbols; $2.54 \mathrm{mV} /$ $\mathrm{mmHg}$ ). For all averaged patch responses (magenta symbols), the response was intermediate with standard errors the largest, as predicted from the categorization results.

In Fig. 9, we plot average NLC for the negative initial $\mathrm{V}_{\mathrm{h}}$ group. Figure $9 \mathrm{~A}$ shows results at $0 \mathrm{mmHg}$ and Fig. 9B shows results at $-10 \mathrm{mmHg}$. Differences are apparent in the frequency responses, with Fig. 9C highlighting the influence of increasing membrane tension on the intersection frequency, $\mathrm{F}_{\mathrm{is}}$. Increasing tension induces a shift in $F_{\text {is }}$ to higher frequencies. For $0 \mathrm{mmHg}, \mathrm{F}_{\text {is }}$ is $11 \mathrm{kHz}$, whereas $-10 \mathrm{mmHg}$ pressure shifts $\mathrm{F}_{\text {is }}$ to $16.2 \mathrm{kHz}$.

A similar analysis is shown in Fig. 10 for the positive initial $V_{h}$ group. Surprisingly, in this case, increasing tension induces a shift in $\mathrm{F}_{\mathrm{is}}$ to lower frequencies. The results for both groups are summarized in Fig. 11A. This plot shows that the two initial $\mathrm{V}_{\mathrm{h}}$ group responses converge towards a common $\mathrm{F}_{\mathrm{is}}$ at $-10 \mathrm{mmHg}$, namely about $16 \mathrm{kHz}$. It may not be a coincidence that this pressure value is close to the turgor pressure $(\sim 1 \mathrm{kPa})$ of the native $\mathrm{OHC}^{38}$. Finally, in Fig. 11B we plot the absolute magnitude of NLC for the two initial $\mathrm{V}_{\mathrm{h}}$ groups. The negative group shows a very similar frequency response regardless of tension, and is in line with our previous observation on the immutable frequency response of the absolute magnitude of complex NLC during tension changes ${ }^{7}$. However, the positive group shows altered frequency responses, with increases in tension slowing the frequency response. Our data thus indicate an interaction between initial prestin state and tension sensitivity.

\section{Discussion}

$\mathrm{OHC}$ NLC is frequency dependent and low pass. We first demonstrated this in guinea pig OHCs using AC voltage chirp stimuli to assess the real component of complex NLC nearly 30 years ago ${ }^{13}$. Over the years this has been confirmed across species ${ }^{9,39-42}$. Indeed, combining measures across a number of studies, we arrived at a collective estimate of NLC (absolute magnitude) frequency response that led us to suggest that prestin activity could not drive eM to sufficiently influence cochlear mechanics at very high acoustic frequencies $(>50 \mathrm{kHz})$, where cochlear amplification is expected to work best ${ }^{7}$. Recently, Rabbitt ${ }^{21}$, expanding on earlier modelling investigations $s^{16,43-45}$, has modelled OHC NLC as a piezoelectric process whose imaginary complex component takes on special significance, namely, signifying power output associated with prestin charge displacement. He modelled that as the real component of NLC decreases across frequency, the imaginary component increases-a frequency-dependent, reciprocal trade-off in magnitudes. Thus, he suggested that dielectric loss in voltage-driven prestin charge movement is indicative of considerable influence at high frequencies, thereby overriding our suggestions that prestin is limited in its high frequency effectiveness ${ }^{7,46}$.

Here we find experimentally that prestin's complex NLC displays a partially reciprocal trade-off between real and imaginary components across interrogating frequency that follows predictions based on the PZE model 
A
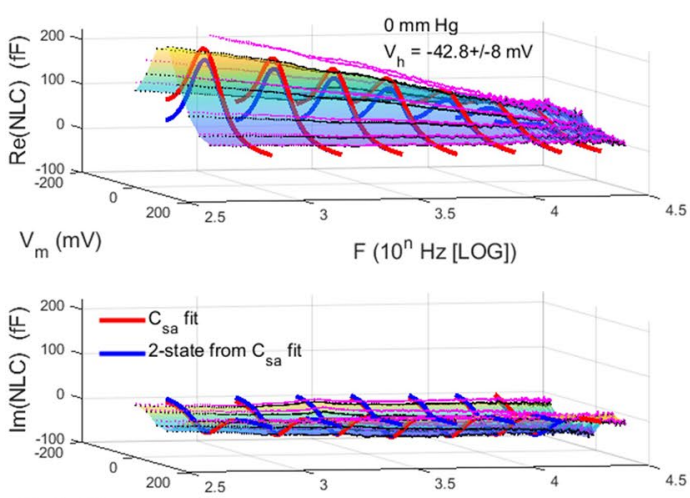

$V_{m}(m V)$

$F\left(10^{n} \mathrm{~Hz}[L O G]\right)$

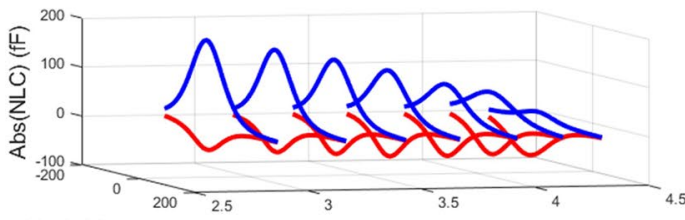

$\mathrm{V}_{\mathrm{m}}(\mathrm{mV}) \quad \mathrm{F}\left(10^{\mathrm{n}} \mathrm{Hz}[\mathrm{LOG}]\right)$

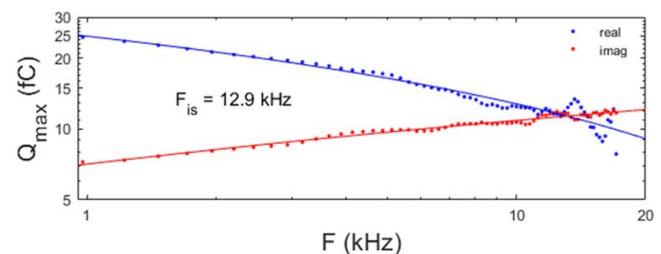

C
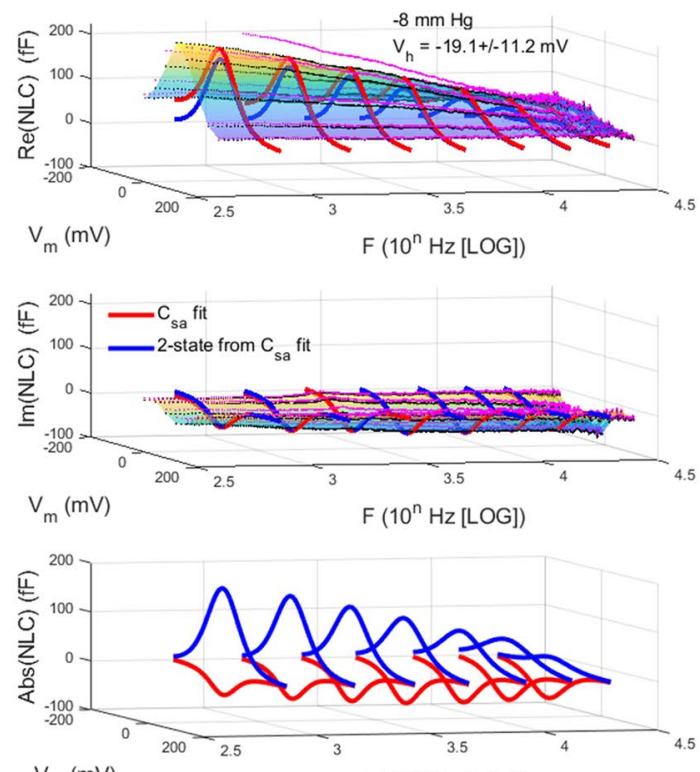

$$
\mathrm{V}_{\mathrm{m}}(\mathrm{mV}) \quad \mathrm{F}\left(10^{\mathrm{n}} \mathrm{Hz}[\text { [LOG] })\right.
$$

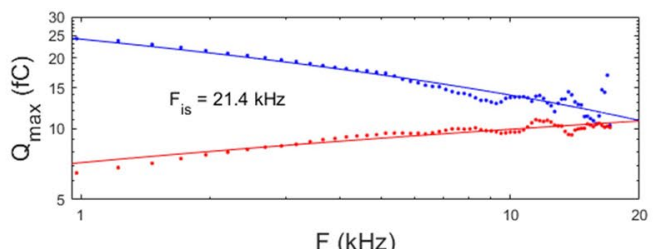

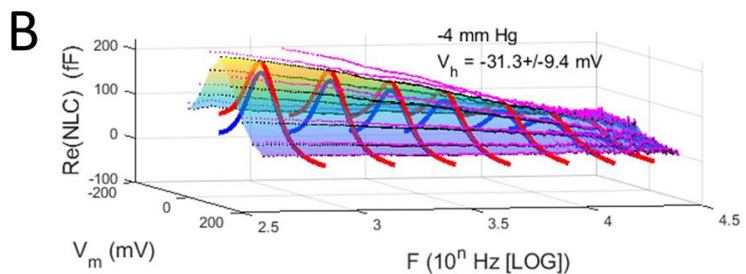
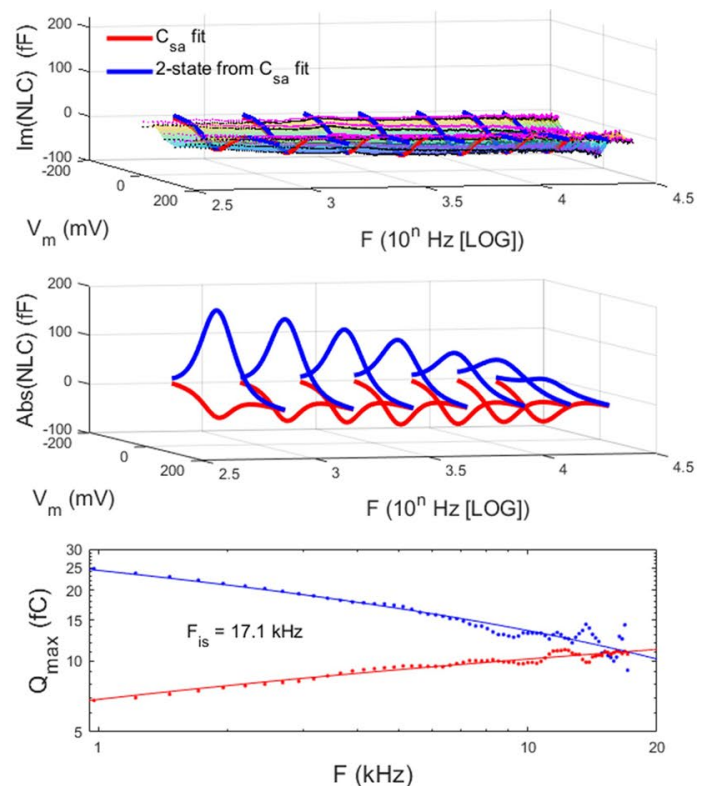

$\mathrm{D}$
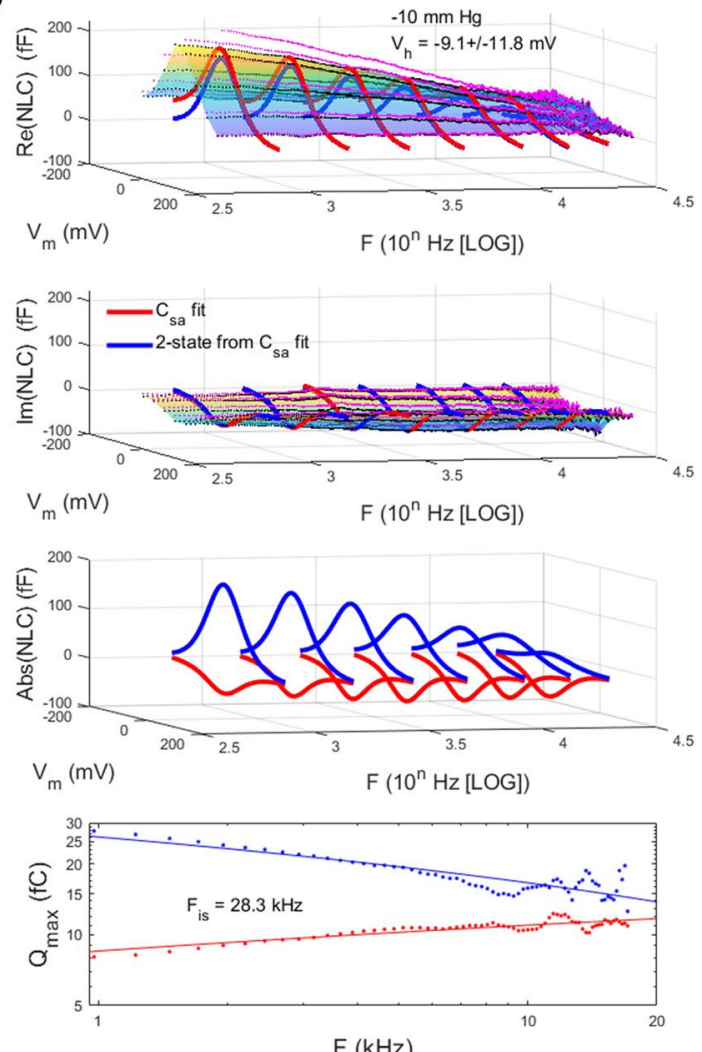

$\mathrm{F}(\mathrm{kHz})$

Figure 7. Effects of membrane tension on complex NLC. Pipette pressure was set to (A) $0 \mathrm{mmHg},(\mathbf{B})-$ $4 \mathrm{mmHg},(\mathbf{C})-8 \mathrm{mmHg}$, and (D) $-10 \mathrm{mmHg}$. Responses are characterized as in Fig. 1 legend. The bottom panels show that $\mathrm{F}_{\text {is }}$ increases as tension increases. 


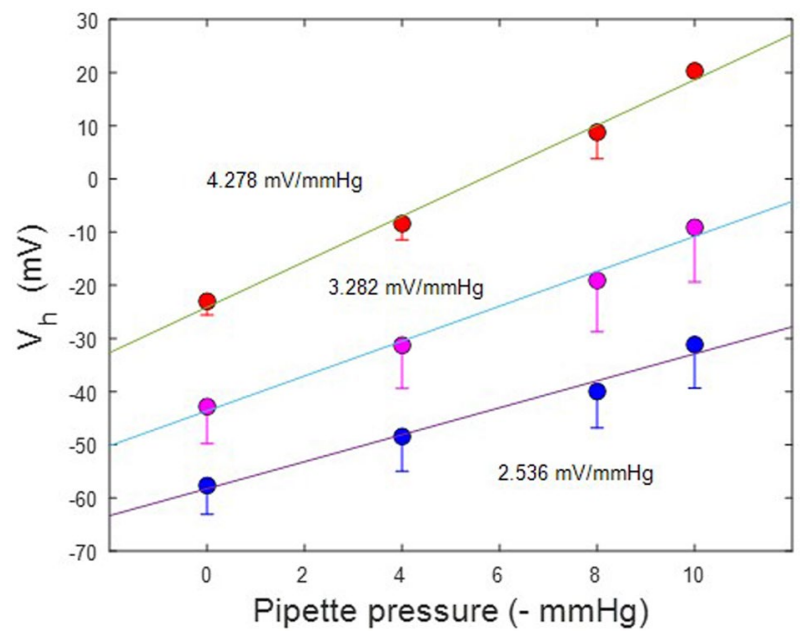

Figure 8. Shift in $V_{h}$ due to tension versus initial $V_{h}$. This plot shows that sensitivity to tension differs depending on initial $V_{h}$. Upon categorization of initial $V_{h}$ with values greater than and less than $-30 \mathrm{mV}$, differences in sensitivity to tension are exposed. In those patches with initial $\mathrm{V}_{\mathrm{h}}$ greater than $-30 \mathrm{mV}$ (red symbols; $-23.0 \pm 2.6 \mathrm{mV} ; 4.28 \mathrm{mV} / \mathrm{mmHg} ; \mathrm{n}=3$ ), sensitivity is nearly double that of the group with initial $\mathrm{V}_{\mathrm{h}}$ less than -30 (blue symbols; $-57.6 \pm 5.4 \mathrm{mV} ; 2.536 \mathrm{mV} / \mathrm{mmHg} ; \mathrm{n}=4$ ). For comparison, the relationship for all average patches is shown (magenta symbols), where a sensitivity to tensions is $3.3 \mathrm{mV} / \mathrm{mmHg}$. This latter relationship is derived from data in Fig. 7. Linear fits provide the slope sensitivity.

A
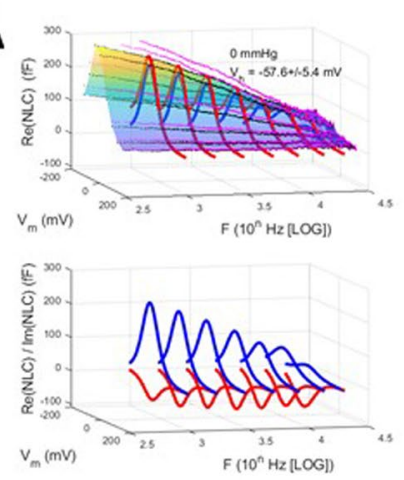

B
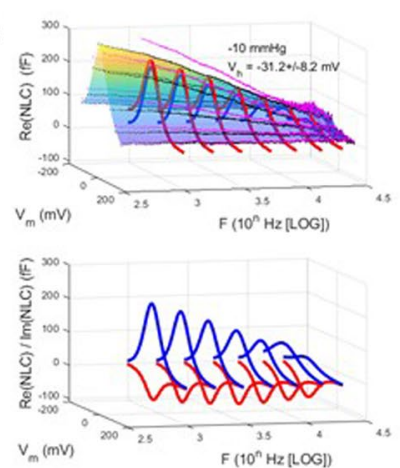
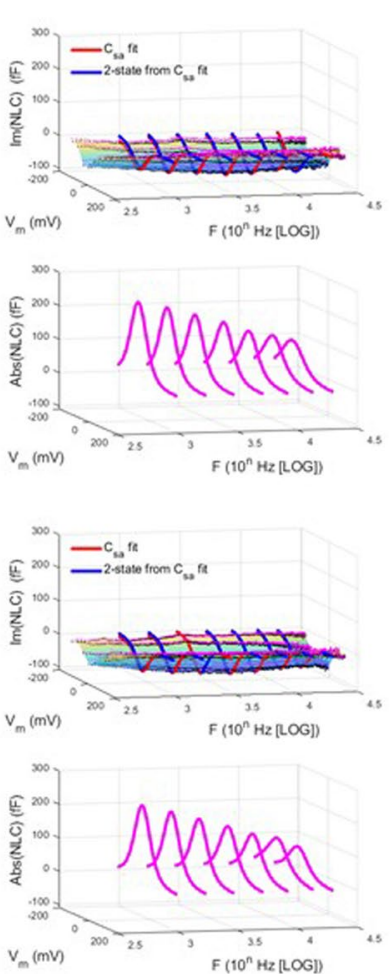

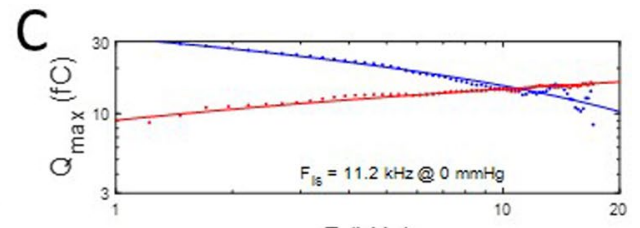

$\mathrm{F}(\mathrm{kHz})$

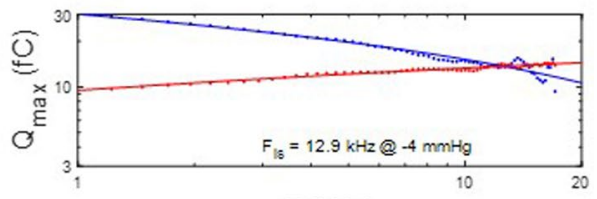

$\mathrm{F}(\mathrm{kHz})$

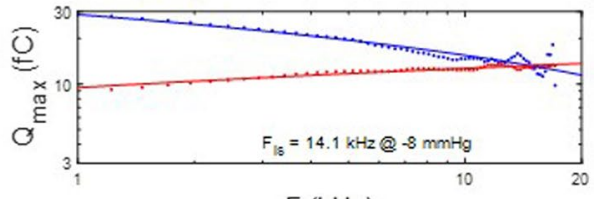

$\mathrm{F}(\mathrm{kHz})$

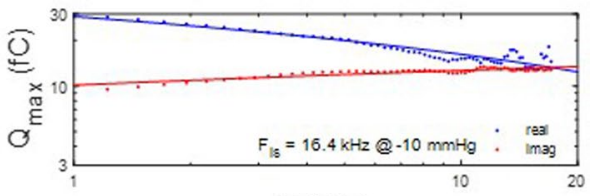

$\mathrm{F}(\mathrm{kHz})$

Figure 9. Membrane tension effects on the frequency response of complex capacitance for negative initial $\mathrm{V}_{\mathrm{h}}$. (A) $0 \mathrm{mmHg}$, (B) $-10 \mathrm{mmHg}$. Clear changes, especially in the imaginary component, are noted between the two tensions. These changes are highlighted in $(\mathbf{C})$ where the intersection frequency, $\mathrm{F}_{\text {is }}$, is found to increase with increases in tension. A shift from 11 to $16.2 \mathrm{kHz}$ occurs between the two extremes.

of prestin ${ }^{21}$. However, we find that similar behavior is found with a simple 2-state kinetic model that is not piezoelectric, but solely voltage-dependent. Prior to Rabbitt's work ${ }^{21}$, Iwasa ${ }^{47}$ developed a 2-state PZE model of prestin which explicitly incorporates tension dependence of prestin's conformational states. Our 2-state model, which evidences real and imaginary parts of NLC, is dependent only on voltage, not tension, and thus is not piezoelectric. Thus, this type of complex behavior that we observe in our biophysical data is not necessarily due 
A
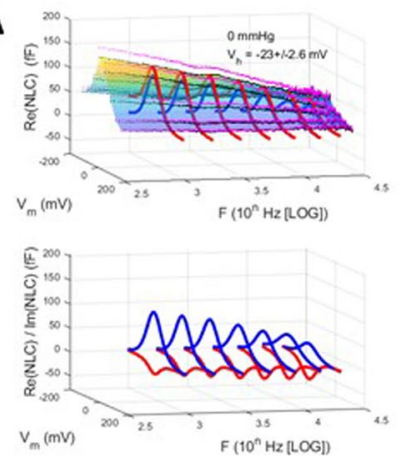

B
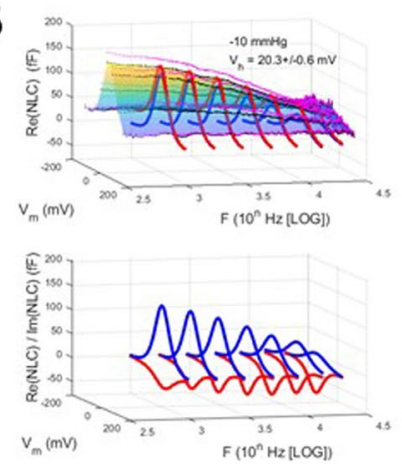
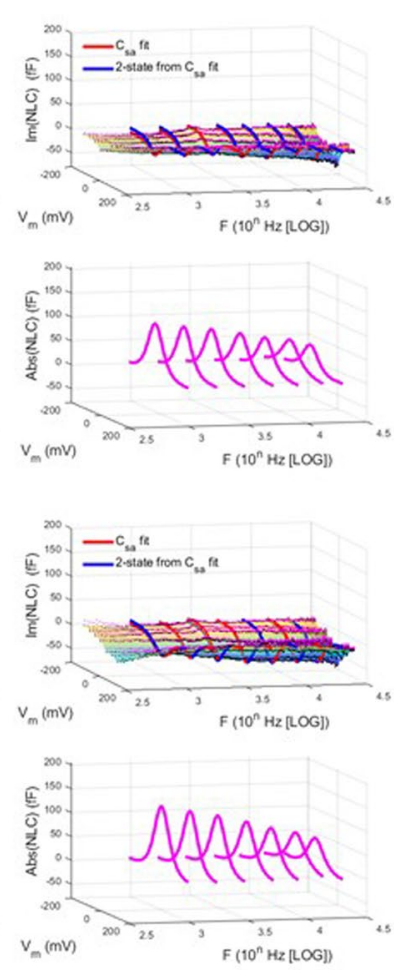
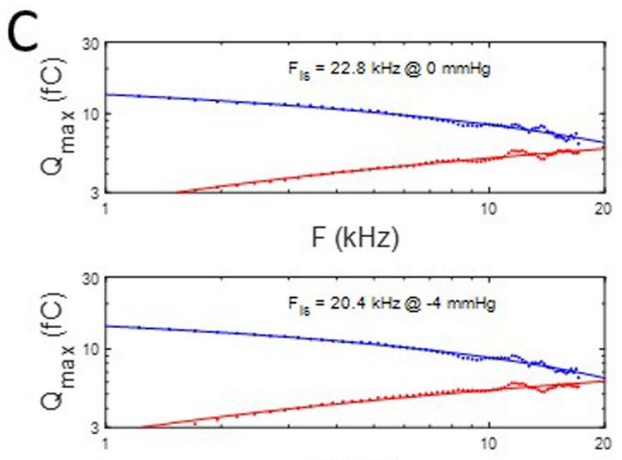

$\mathrm{F}(\mathrm{kHz})$
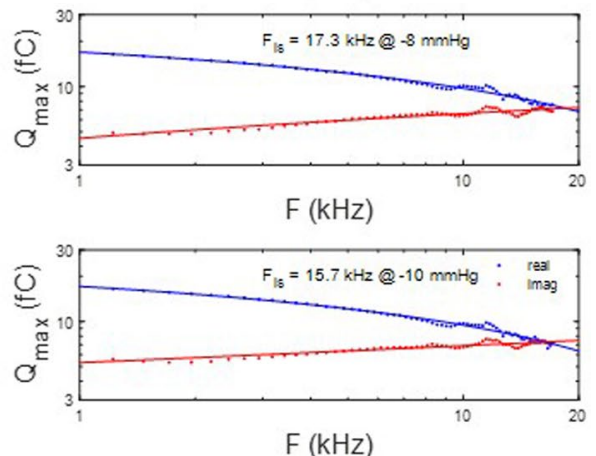

Figure 10. Membrane tension effects on the frequency response of complex capacitance for positive initial $\mathrm{V}_{\mathrm{h}}$. (A) $0 \mathrm{mmHg}$, (B) - $10 \mathrm{mmHg}$. Clear changes, especially in the imaginary component, are noted between the two tensions. These changes are highlighted in $(\mathbf{C})$ where the intersection frequency, $\mathrm{F}_{\mathrm{is}}$, is found to decrease with increases in tension. A shift from 22.1 to $16.8 \mathrm{kHz}$ occurs between the two extremes.

to piezoelectricity in prestin, and whether other predictions of the PZE model correspond to the observed prestin behavior that we find remains to be seen. To be sure, we are not claiming that prestin is not piezoelectric-like, since we have substantially contributed to the experimental evidence that it is, but only that non PZE models can generate real and imaginary components of NLC.

For decades, we and others ${ }^{9,13,39,44,48}$ have suggested that NLC and eM behavior are governed by the conformational kinetics of OHC motor (prestin) transitions, in line with traditional biophysical concepts of voltagedependent protein behavior. Indeed, when we initially realized that prestin kinetics were influenced by chloride ions ${ }^{49,50}$, we suggested that delays introduced by stretched exponential transition rates in prestin would impact the phase of eM. Subsequently, we found that an eM phase lag (re voltage) develops across frequency and this could be influenced by chloride anions ${ }^{51}$. It is likely that the frequency dependent imaginary component of NLC we observe here correlates with that out of phase mechanical response. Thus, we agree with Rabbitt ${ }^{21}$ that the imaginary component of NLC may have special significance in prestin function, regardless of whether prestin works as a piezoelectric device or not. Nevertheless, our data show that absolute magnitude of NLC (representing total charge, including in and out of phase components) decreases as a power function of frequency, being $40 \mathrm{~dB}$ down at $77 \mathrm{kHz}^{7}$. This is a minuscule fraction of voltage-driven prestin activity that exists at low frequencies. Based on PZE models ${ }^{16,21,47}$, mechanical loads can influence the frequency response of prestin. We agree that NLC and eM are susceptible to mechanical load, but the frequency response of whole-cell eM is slower than that of NLC, and likely corresponds to both cellular (external) and molecular (intrinsic) load components ${ }^{8}$. Thus, with minimal influence of external loads, we view our macro-patch data as providing the best estimate of prestin's intrinsic conformational switching limit (including all influential molecular impedances), as originally espoused by Gale and Ashmore ${ }^{39}$.

Characteristics of OHC NLC have been known for some time to depend on initial conditions; for example, $\mathrm{V}_{\mathrm{h}}$ depends on initial holding potential and anion binding is state-dependent ${ }^{33,52,53}$. In an effort to determine whether complex NLC shows sensitivity to initial conditions, we categorized our data into classes that had initial $\mathrm{V}_{\mathrm{h}}$ values above and below $-30 \mathrm{mV}$. We found effects of initial conditions on the frequency response of real and imaginary components, where a metric of this relationship, $\mathrm{F}_{\mathrm{is}}$, the intersection frequency of real and imaginary magnitude components varies with initial $V_{h}$. As initial $V_{h}$ shifts positively, $F_{\text {is }}$ increases nonlinearly (see Fig. 6).

We also found initial $V_{h}$ effects on the influence of static membrane tension on $F_{i s}$. Whereas the group with initial $V_{h}$ positive to $-30 \mathrm{mV}$ shows a decrease in $F_{\text {is }}$ with increasing membrane tension, the opposite is found in the group with initial $\mathrm{V}_{\mathrm{h}}$ negative to $-30 \mathrm{mV}$. At $-10 \mathrm{mmHg}(1.22 \mathrm{kPa})$, each group's $\mathrm{F}_{\text {is }}$ intersects near $16 \mathrm{kHz}$ (see Fig. 11A). Interestingly, this pressure value is close to the turgor pressure (about $1 \mathrm{kPa}$ ) of the native cylindrical $\mathrm{OHC}^{38}$.

Lastly, we found that the group with initial $\mathrm{V}_{\mathrm{h}}$ negative to $-30 \mathrm{mV}$ shows little variation in the frequency response of the absolute magnitude of NLC with membrane tension, similar to what we observed previously ${ }^{7}$. 

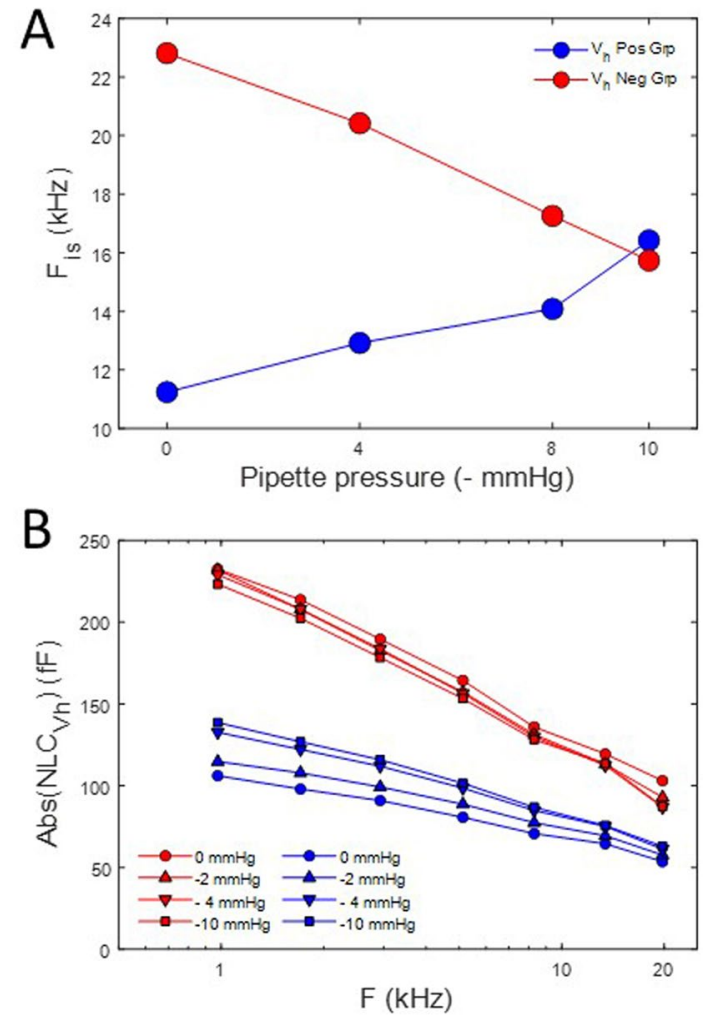

Figure 11. Summary of initial $V_{h}$ influence on tension effects. (A) The direction of change in $F_{\text {is }}$ is dependent on initial $\mathrm{V}_{\mathrm{h}}$. Analyses were made with stray capacitance removal using either the $+160 \mathrm{mV}$ (circles) or $-160 \mathrm{mV}$ (asterisks) holding admittance. Results are identical, as expected. The two initial $\mathrm{V}_{\mathrm{h}}$ group responses converge until a common $\mathrm{F}_{\text {is }}$ vs. tension is reached at $-10 \mathrm{mmHg}$, namely about $16 \mathrm{kHz}$. (B) Plot of the absolute magnitude of NLC for the two initial $\mathrm{V}_{\mathrm{h}}$ groups. The negative group shows a very similar frequency response regardless of tension, but the positive group shows altered frequency responses, with increases in tension slowing the frequency response.

However, the frequency response of the group with initial $V_{h}$ positive to $-30 \mathrm{mV}$ shows a decrease in the frequency response as membrane tension increases. From all these observations, it appears that the frequency response/magnitude of the imaginary component of NLC is mainly sensitive to initial $\mathrm{V}_{\mathrm{h}}$. Though adherence to a minimum phase system response might predict a complimentary relationship between real and imaginary components, viscoelastic non-minimum phase systems are known in biology ${ }^{54,55}$. Consequently, our data indicate that the state of prestin, characterized by initial $\mathrm{V}_{\mathrm{h}}$, influences frequency relationships between components of complex NLC, and thus, according to Rabbitt ${ }^{21}$, the power output of prestin activity. Nevertheless, changes in the frequency response are relatively small with our perturbations.

The fact that the individual components of complex NLC are differentially susceptible indicates that frequency-dependent phase differences occur between elements of sensor charge movement, possibly indicating that widely distributed sensor charge residues ${ }^{56}$ may independently interact within local intra-protein, nanoenvironments. Supporting this view, recent cryo-EM determined structural features of the mechano-sensitive channel MscS point to differential interactions of the protein with lipids surrounding and within the protein itself $^{57}$. Thus, the study of complex NLC of our sensor charge residue mutations ${ }^{56}$ in HEK cell macro-patches, may assign particular residues responsible for the complex component responses. For example, we may find that certain mutations may remove the imaginary component without altering the real component.

As we noted above, there are several factors that influence $V_{h}$. In the case of membrane tension, what could govern $\mathrm{V}_{\mathrm{h}}$ ? There are numerous descriptions of membrane proteins/channels that are influenced by membrane tension $^{58}$, with some possessing voltage-dependence; yet, interestingly, none have been deemed piezoelectric, per se. Rather, standard biophysical influences of load on a protein's conformational state are espoused. For example, some $\mathrm{Kv}$ channels are voltage and tension sensitive, and simple models where changes in the equilibrium constant for channel opening, or equivalently the ratio of forward to backward transition rates, can account for tension effects on channel activity ${ }^{59}$. What other influences could there be in the absence of direct PZE effects or direct biophysical mechanical influences on prestin?

Several ideas come to mind. Perhaps the degree of cooperativity among prestin units alters with membrane tension. We recently provided evidence for negative cooperativity in prestin that was related to the density of prestin within the membrane ${ }^{60}$. Additionally, we previously modelled initial voltage influences on NLC as resulting from $\mathrm{OHC}$ molecular motor-motor (prestin) interactions ${ }^{33}$. For Kv 7.4 channels, cooperativity has been found to impart mechanical sensitivity to the channel in $\mathrm{OHCs}^{61}$. Another possibility is the presence of a conductive 
element influencing the interaction between voltage sensor charge and the membrane field, where that element alters with tension. Under this circumstance, the voltage sensed by the prestin charge may change during alterations in tension, leading to apparent $\mathrm{V}_{\mathrm{h}}$ shifts relative to voltage clamp commands. Could this be a viscoelastic coupled conductive element, thus providing time dependent changes in voltage sensed? We have observed multi-exponential time-dependent behavior in NLC at fixed voltages ${ }^{32,33}$. Is $\mathrm{G}_{\text {metL }}$, the tension-dependent prestin leakage conductance we have identified ${ }^{62}$, the conductive component? We are currently testing complex NLC in prestin mutants that have reduced $\mathrm{G}_{\mathrm{met}}$ conductance.

Finally, the shift in $\mathrm{V}_{\mathrm{h}}$ could additionally result from changes in the membrane surface charge or dipole potential that may accompany changes in membrane tension. Warshaviak et al. ${ }^{63}$ have found that physiologically relevant changes in membrane tension could shift that potential by tens of millivolts. Such effects could produce changes in the distribution of voltage-dependent protein conformations that would be evident as altered $V_{h}$, despite effective voltage clamp. Interestingly, changes in temperature could conceivably alter the membrane dipole (in addition to many other properties of the membrane), since electrical breakdown in membrane is temperature dependent ${ }^{64}$. Thus, our observation of shifts in $\mathrm{V}_{\mathrm{h}}$ due to temperature ${ }^{29-31}$ could also be independent of direct action on prestin. Of course, in this case of these "charge screening" effects, alterations in transition rates would not underlie $V_{h}$ shifts, so this is unlikely to fully account for prestin's tension responsiveness since we do find changes in the frequency response of real and imaginary components of complex NLC. Hence, transition rates are indeed altered.

To summarize our observations, we find that prestin's complex NLC displays partial reciprocal trade-offs in real and imaginary component magnitude across frequency. The trade-off is prestin state-dependent in that the frequency response of the components alter with initial $\mathrm{V}_{\mathrm{h}}$ and membrane tension. However, these observations do not nullify our initial observations ${ }^{7,46}$ that absolute complex NLC, signifying total charge moved, decreases precipitously as a power function of frequency; thus, prestin charge displacement that correlates with electromotility ${ }^{9}$ is expected to have limited physiological influence at very high frequencies in excess of $50 \mathrm{kHz}$.

Some final comments on the OHC's ability to drive cochlear amplification at very high frequencies in a hypothetical cycle-by-cycle manner are in order. The membrane RC filter, which limits high frequency AC receptor potential generation, has been considered a potential problem for decades ${ }^{65,66}$, and while there are a number of publications that have suggested that the problem could be alleviated ${ }^{17,67-69}$, none have been directly tested in vivo. Thus, the RC problem may remain, and indeed arguments have been made for its effect on eM measured in vivo ${ }^{10}$. Both the potential RC problem and the low-pass nature of prestin charge movement pose serious obstacles for proposed theoretical mechanisms that offer to circumvent an OHC speed limit ${ }^{8}$.

\section{Methods}

Detailed methods, including specifics of our voltage chirp stimulus protocol and extraction of complex NLC from patch admittance, are available in Refs. ${ }^{746}$. Briefly, extracellular solution was (in mM): $\mathrm{NaCl} 100, \mathrm{TEA}-\mathrm{Cl} 20, \mathrm{CsCl}$ $20, \mathrm{CoCl}_{2} 2, \mathrm{MgCl}_{2} 1, \mathrm{CaCl}_{2}$, Hepes 10, $\mathrm{pH}$ 7.2. Experiments were performed at room temperature. Extracellular solution was in the patch pipette. On-cell macro-patches on the guinea pig OHC lateral membrane were made near the middle of the cylindrical cell, where prestin resides at high density ${ }^{70,71}$. Pipette inner tip size was about $3.5 \mu \mathrm{m}$, series resistance $\left(\mathrm{R}_{\mathrm{s}}\right)$ estimated to be below $1 \mathrm{M} \Omega$, and seals at $0 \mathrm{mV}$ near $5 \mathrm{G} \Omega$ (see Ref. ${ }^{7}$ ). Under these conditions, $\mathrm{R}_{\mathrm{s}}$ effects are minimal ${ }^{7}$ and were not corrected for in this report. Furthermore, in this report we make relative assessments of NLC among groups of macro-patches. Chirp voltage stimuli (10 mV pk) were superimposed on step holding potentials from -160 to $+160 \mathrm{mV}$, in $40 \mathrm{mV}$ increments. As detailed previously ${ }^{7}$, the FFT derived admittance at $+160 \mathrm{mV}$, where NLC is absent, is subtracted from admittance at all other step potentials, thereby removing stray capacitance. Additionally, because we focus on effects of $V_{h}$ on complex NLC behavior, we confirmed that subtractions for stray capacitance using either +160 or $-160 \mathrm{mV}$ responses give the exact same Boltzmann fits, as expected since stray capacitance will not influence prestin charge movement/ NLC. A residual DC nonlinear conductance was also removed. DC conductance was determined from the DC component of FFT current response at each stepped voltage. Conductance, $\Delta \mathrm{I}(0) / \Delta \mathrm{V}_{\text {hold }}$, was gauged by spline interpolating between each step voltage response, and differentiating digitally. Since, such an approach can have untoward effects at the end point voltage extremes, we fit the conductance linearly between 0 and $396 \mathrm{~Hz}$ (conductance at each frequency determined from the real components), and used the resultant fitted conductance at zero $\mathrm{Hz}$ for subtraction of the real components of admittance across frequencies. Figure 3 illustrates, in a simple 2-state kinetic model with solely voltage-dependent transition rates, the necessity of such nonlinear DC conductance subtractions to validly extract the imaginary component of NLC at low frequencies. The model is fully described in the "Results" Section. Patch membrane tension was imposed by changing pipette pressure. All data collection and analyses were performed with the software programs jClamp (www.scisoftco.com) and Matlab (www.mathworks.com). All means and standard errors (SE) are from individually analyzed patch data. Plots were made in Matlab.

In order to extract Boltzmann parameters, capacitance-voltage data were fit to the first derivative of a twostate Boltzmann function ${ }^{13,39}$, with an component of capacitance that characterizes sigmoidal changes in specific membrane capacitance ${ }^{24,72}$. We refer to this as the " $\mathrm{C}_{\mathrm{sa}}$ fit" in text and figures.

$$
C_{m}=N L C+C_{s a}+C_{\operatorname{lin}}=Q_{\max } \frac{\mathrm{ze}}{k_{B} T} \frac{b}{(1+b)^{2}}+C_{s a}+C_{\text {lin }},
$$

where $b=\exp \left(-z e \frac{V_{m}-V_{h}}{k_{B} T}\right), C_{s a}=\frac{\Delta C_{s a}}{\left(1+b^{-1}\right)}$.

$\mathrm{Q}_{\max }$ is the maximum nonlinear charge moved, $\mathrm{V}_{\mathrm{h}}$ is voltage at peak capacitance or equivalently, at halfmaximum charge transfer, $\mathrm{V}_{\mathrm{m}}$ is membrane potential, $z$ is valence, $\mathrm{C}_{\text {lin }}$ is linear membrane capacitance, e is electron charge, $k_{B}$ is Boltzmann's constant, and $\mathrm{T}$ is absolute temperature. $\mathrm{C}_{\mathrm{sa}}$ is a component of capacitance that 
characterizes sigmoidal changes in specific membrane capacitance, with $\Delta \mathrm{C}_{\mathrm{sa}}$ referring to the maximal change at very negative voltages ${ }^{24,72}$.

Qmax is the maximum nonlinear charge moved, Vh is voltage at peak capacitance or equivalently, at halfmaximum charge transfer, $\mathrm{Vm}$ is membrane potential, $\mathrm{z}$ is valence, Clin is linear membrane capacitance, e is electron charge, $\mathrm{kB}$ is Boltzmann's constant, and T is absolute temperature. Csa is a component of capacitance that characterizes sigmoidal changes in specific membrane capacitance, with $\Delta$ Csa referring to the maximal change at very negative voltages ${ }^{24,72}$.

For some data, a power fit as a function of frequency $(f)$ was performed ${ }^{46,73}$.

$$
C(f)=C_{0}+a \times f^{b},
$$

where $\mathrm{C}_{0}$ is the asymptotic component, and $a$ and $b$ control the frequency response.

Received: 24 March 2021; Accepted: 21 July 2021

Published online: 09 August 2021

\section{References}

1. Santos-Sacchi, J. \& Dilger, J. P. Whole cell currents and mechanical responses of isolated outer hair cells. Hear. Res. 35, 143-150 (1988).

2. Zheng, J. et al. Prestin is the motor protein of cochlear outer hair cells. Nature 405, 149-155 (2000).

3. Liberman, M. C. et al. Prestin is required for electromotility of the outer hair cell and for the cochlear amplifier. Nature 419, 300-304 (2002)

4. Frank, G., Hemmert, W. \& Gummer, A. W. Limiting dynamics of high-frequency electromechanical transduction of outer hair cells. Proc. Natl. Acad. Sci. U.S.A. 96, 4420-4425 (1999).

5. Nuttall, A. L., Zheng, J., Ren, T. \& de Boer, E. Electrically evoked otoacoustic emissions from apical and basal perilymphatic electrode positions in the guinea pig cochlea. Hear. Res. 152, 77-89. https://doi.org/10.1016/s0378-5955(00)00238-0 (2001).

6. Drexl, M., Lagarde, M. M., Zuo, J., Lukashkin, A. N. \& Russell, I. J. The role of prestin in the generation of electrically evoked otoacoustic emissions in mice. J. Neurophysiol. 99, 1607-1615 (2008).

7. Santos-Sacchi, J. \& Tan, W. Complex nonlinear capacitance in outer hair cell macro-patches: Effects of membrane tension. Sci. Rep. 10, 6222. https://doi.org/10.1038/s41598-020-63201-6 (2020).

8. Santos-Sacchi, J., Iwasa, K. H. \& Tan, W. Outer hair cell electromotility is low-pass filtered relative to the molecular conformational changes that produce nonlinear capacitance. J. Gen. Physiol. 151, 1369-1385. https://doi.org/10.1085/jgp.201812280 (2019).

9. Santos-Sacchi, J. \& Tan, W. The frequency response of outer hair cell voltage-dependent motility is limited by kinetics of prestin. J. Neurosci. 38, 5495-5506. https://doi.org/10.1523/JNEUROSCI.0425-18.2018 (2018).

10. Vavakou, A., Cooper, N. P. \& van der Heijden, M. The frequency limit of outer hair cell motility measured in vivo. Elife https:// doi.org/10.7554/eLife.47667 (2019)

11. van der Heijden, M. \& Versteegh, C. P. Energy flux in the cochlea: Evidence against power amplification of the traveling wave. J. Assoc. Res. Otolaryngol. 16, 581-597. https://doi.org/10.1007/s10162-015-0529-5 (2015).

12. Ashmore, J. F. Forward and reverse transduction in the mammalian cochlea. Neurosci. Res. Suppl. 12, S39-S50 (1990).

13. Santos-Sacchi, J. Reversible inhibition of voltage-dependent outer hair cell motility and capacitance. J. Neurosci. 11, 3096-3110 (1991).

14. Kilic, G. \& Lindau, M. Voltage-dependent membrane capacitance in rat pituitary nerve terminals due to gating currents. Biophys. J. 80, 1220-1229. https://doi.org/10.1016/S0006-3495(01)76098-5 (2001).

15. Iwasa, K. H. Effect of stress on the membrane capacitance of the auditory outer hair cell. Biophys. J. 65, 492-498 (1993).

16. Dong, X. X., Ospeck, M. \& Iwasa, K. H. Piezoelectric reciprocal relationship of the membrane motor in the cochlear outer hair cell. Biophys. J. 82, 1254-1259 (2002).

17. Kakehata, S. \& Santos-Sacchi, J. Membrane tension directly shifts voltage dependence of outer hair cell motility and associated gating charge. Biophys. J. 68, 2190-2197 (1995).

18. Gale, J. E. \& Ashmore, J. F. Charge displacement induced by rapid stretch in the basolateral membrane of the guinea-pig outer hair cell. Proc. R. Soc. Lond. B. Biol. Sci. 255, 243-249 (1994).

19. Gale, J. E. \& Ashmore, J. F. The outer hair cell motor in membrane patches. Pflugers Arch. 434, 267-271 (1997).

20. Santos-Sacchi, J. Functional motor microdomains of the outer hair cell lateral membrane. Pflugers Arch. 445, 331 (2002).

21. Rabbitt, R. D. The cochlear outer hair cell speed paradox. Proc. Natl. Acad. Sci. U.S.A. 117, 21880-21888. https://doi.org/10.1073/ pnas.2003838117 (2020).

22. Bezanilla, F. How membrane proteins sense voltage. Nat. Rev. Mol. Cell Biol. 9, 323-332 (2008).

23. Fernandez, J. M., Bezanilla, F. \& Taylor, R. E. Distribution and kinetics of membrane dielectric polarization. II. Frequency domain studies of gating currents. J. Gen. Physiol 79, 41-67 (1982).

24. Santos-Sacchi, J. \& Navarrete, E. Voltage-dependent changes in specific membrane capacitance caused by prestin, the outer hair cell lateral membrane motor. Pflugers Arch. 444, 99-106 (2002).

25. Kalinec, F., Holley, M. C., Iwasa, K. H., Lim, D. J. \& Kachar, B. A membrane-based force generation mechanism in auditory sensory cells. Proc. Natl. Acad. Sci. U.S.A. 89, 8671-8675 (1992).

26. Rybalchenko, V. \& Santos-Sacchi, J. Anion control of voltage sensing by the motor protein prestin in outer hair cells. Biophys. J. 95, 4439-4447 (2008).

27. Santos-Sacchi, J., Song, L., Zheng, J. F. \& Nuttall, A. L. Control of mammalian cochlear amplification by chloride anions. J. Neurosci. 26, 3992-3998. https://doi.org/10.1523/jneurosci.4548-05.2006 (2006).

28. Oliver, D. et al. Intracellular anions as the voltage sensor of prestin, the outer hair cell motor protein. Science 292, 2340-2343 (2001).

29. Okunade, O. \& Santos-Sacchi, J. IR laser-induced perturbations of the voltage-dependent solute carrier protein SLC26a5. Biophys. J. 105, 1822-1828. https://doi.org/10.1016/j.bpj.2013.09.008 (2013).

30. Meltzer, J. \& Santos-Sacchi, J. Temperature dependence of non-linear capacitance in human embryonic kidney cells transfected with prestin, the outer hair cell motor protein. Neurosci. Lett. 313, 141-144 (2001).

31. Santos-Sacchi, J. \& Huang, G. Temperature dependence of outer hair cell nonlinear capacitance. Hear. Res. 116, 99-106 (1998).

32. Santos-Sacchi, J., Navarrete, E. \& Song, L. Fast electromechanical amplification in the lateral membrane of the outer hair cell. Biophys. J. 96, 739-747. https://doi.org/10.1016/j.bpj.2008.10.015 (2009).

33. Santos-Sacchi, J., Kakehata, S. \& Takahashi, S. Effects of membrane potential on the voltage dependence of motility-related charge in outer hair cells of the guinea-pig. J. Physiol. 510(Pt 1), 225-235 (1998).

34. Sfondouris, J., Rajagopalan, L., Pereira, F. A. \& Brownell, W. E. Membrane composition modulates prestin-associated charge movement. J. Biol. Chem. 283, 22473-22481 (2008). 
35. Rajagopalan, L. et al. Tuning of the outer hair cell motor by membrane cholesterol. J. Biol. Chem. 282, 36659-36670 (2007).

36. Corbitt, C., Farinelli, F., Brownell, W. E. \& Farrell, B. Tonotopic relationships reveal the charge density varies along the lateral wall of outer hair cells. Biophys. J. 102, 2715-2724. https://doi.org/10.1016/j.bpj.2012.04.054 (2012).

37. Takahashi, S. \& Santos-Sacchi, J. Non-uniform mapping of stress-induced, motility-related charge movement in the outer hair cell plasma membrane. Pflugers Arch. 441, 506-513 (2001).

38. Ratnanather, J., Brownell, W. \& Popel, A. Mechanical properties of the outer hair cell. In Biophysics of Hair Cell Sensory Systems (eds Duifhuis, H. et al.) 199-206 (World Scientific, 1993).

39. Gale, J. E. \& Ashmore, J. F. An intrinsic frequency limit to the cochlear amplifier. Nature 389, 63-66 (1997).

40. Homma, K., Duan, C., Zheng, J., Cheatham, M. A. \& Dallos, P. The V499G/Y501H mutation impairs fast motor kinetics of prestin and has significance for defining functional independence of individual prestin subunits. J. Biol. Chem. 288, 2452-2463. https:// doi.org/10.1074/jbc.M112.411579 (2013).

41. Albert, J. T. et al. Voltage-sensitive prestin orthologue expressed in zebrafish hair cells. J. Physiol. 580, 451-461 (2007).

42. Ren, T. et al. (eds) Auditory Mechanisms, Processes and Models 231-232 (World Scientific, 2006).

43. Sun, S. X. et al. Voltage and frequency dependence of prestin-associated charge transfer. J. Theor. Biol. 260, 137-144. https://doi. org/10.1016/j.jtbi.2009.05.019 (2009).

44. Iwasa, K. H. Current noise spectrum and capacitance due to the membrane motor of the outer hair cell: Theory. Biophys. J. 73, 2965-2971 (1997).

45. Spector, A. A., Deo, N., Grosh, K., Ratnanather, J. T. \& Raphael, R. M. Electromechanical models of the outer hair cell composite membrane. J. Membr. Biol. 209, 135-152 (2006).

46. Santos-Sacchi, J. \& Tan, W. Voltage does not drive prestin (SLC26a5) electro-mechanical activity at high frequencies where cochlear amplification is best. iScience 22, 392-399. https://doi.org/10.1016/j.isci.2019.11.036 (2019).

47. Iwasa, K. H. A two-state piezoelectric model for outer hair cell motility. Biophys. J. 81, 2495-2506 (2001).

48. Santos-Sacchi, J. Fast outer hair cell motility: How fast is fast? In The Mechanics and Biophysics of Hearing (eds Dallos, P. et al.) 69-75 (Springer, 1990).

49. Song, L. \& Santos-Sacchi, J. Disparities in voltage-sensor charge and electromotility imply slow chloride-driven state transitions in the solute carrier SLC26a5. Proc. Natl. Acad. Sci. U.S.A. https://doi.org/10.1073/pnas.1218341110 (2013).

50. Santos-Sacchi, J. \& Song, L. Chloride anions regulate kinetics but not voltage-sensor Qmax of the solute carrier SLC26a5. Biophys. J. 110,1-11 (2016).

51. Santos-Sacchi, J. \& Song, L. Chloride-driven electromechanical phase lags at acoustic frequencies are generated by SLC26a5, the outer hair cell motor protein. Biophys. J. 107, 126-133. https://doi.org/10.1016/j.bpj.2014.05.018 (2014).

52. Santos-Sacchi, J., Shen, W., Zheng, J. \& Dallos, P. Effects of membrane potential and tension on prestin, the outer hair cell lateral membrane motor protein. J. Physiol. 531, 661-666 (2001).

53. Song, L. \& Santos-Sacchi, J. Conformational state-dependent anion binding in prestin: evidence for allosteric modulation. Biophys. J. 98, 371-376. https://doi.org/10.1016/j.bpj.2009.10.027 (2010).

54. Kobayashi, Y., Okamura, N., Tsukune, M., Fujie, M. G. \& Tanaka, M. Non-minimum phase viscoelastic properties of soft biological tissues. J. Mech. Behav. Biomed. Mater. 110, 103795. https://doi.org/10.1016/j.jmbbm.2020.103795 (2020).

55. Recio-Spinoso, A., Fan, Y. H. \& Ruggero, M. A. Basilar-membrane responses to broadband noise modeled using linear filters with rational transfer functions. IEEE Trans. Biomed. Eng. 58, 1456-1465. https://doi.org/10.1109/Tbme.2010.2052254 (2011).

56. Bai, J. P. et al. Prestin's anion transport and voltage-sensing capabilities are independent. Biophys. J. 96, 3179-3186. https://doi. org/10.1016/j.bpj.2008.12.3948 (2009).

57. Zhang, Y. et al. Visualization of the mechanosensitive ion channel MscS under membrane tension. Nature 590, 509-514. https:// doi.org/10.1038/s41586-021-03196-w (2021).

58. Jin, P., Jan, L. Y. \& Jan, Y. N. Mechanosensitive ion channels: Structural features relevant to mechanotransduction mechanisms. Annu. Rev. Neurosci. 43, 207-229. https://doi.org/10.1146/annurev-neuro-070918-050509 (2020).

59. Schmidt, D., del Marmol, J. \& MacKinnon, R. Mechanistic basis for low threshold mechanosensitivity in voltage-dependent K+ channels. Proc. Natl. Acad. Sci. U.S.A. 109, 10352-10357. https://doi.org/10.1073/pnas.1204700109 (2012).

60. Zhai, F. et al. Maturation of voltage-induced shifts in SLC26a5 (prestin) operating point during trafficking and membrane insertion. Neuroscience 431, 128-133. https://doi.org/10.1016/j.neuroscience.2020.02.003 (2020).

61. Perez-Flores, M. C. et al. Cooperativity of Kv7.4 channels confers ultrafast electromechanical sensitivity and emergent properties in cochlear outer hair cells. Sci. Adv. 6, 1104. https://doi.org/10.1126/sciadv.aba1104 (2020).

62. Bai, J. P. et al. Current carried by the Slc26 family member prestin does not flow through the transporter pathway. Sci. Rep. 7, 46619. https://doi.org/10.1038/srep46619 (2017).

63. Warshaviak, D. T., Muellner, M. J. \& Chachisvilis, M. Effect of membrane tension on the electric field and dipole potential of lipid bilayer membrane. Biochim. Biophys. Acta 2608-2617, 2011. https://doi.org/10.1016/j.bbamem.2011.06.010 (1808).

64. Gneno, R., Azzar, G., Got, R. \& Roux, B. Temperature and external electric field influence in membrane permeability of Babesia infected erythrocytes. Int. J. Biochem. 19, 1069-1073. https://doi.org/10.1016/0020-711x(87)90308-9 (1987).

65. Santos-Sacchi, J. Asymmetry in voltage-dependent movements of isolated outer hair cells from the organ of Corti. J. Neurosci. 9, 2954-2962 (1989).

66. Housley, G. D. \& Ashmore, J. F. Ionic currents of outer hair cells isolated from the guinea-pig cochlea. J. Physiol. (Lond.) 448, 73-98 (1992).

67. Spector, A. A., Brownell, W. E. \& Popel, A. S. Effect of outer hair cell piezoelectricity on high-frequency receptor potentials. J. Acoust. Soc. Am. 113, 453-461 (2003).

68. Ospeck, M., Dong, X. X., Fang, J. \& Iwasa, K. H. Electromotility in outer hair cells: A supporting role for fast potassium conductance. ORL J. Otorhinolaryngol. Relat. Spec. 68, 373-377. https://doi.org/10.1159/000095280 (2006).

69. Johnson, S. L., Beurg, M., Marcotti, W. \& Fettiplace, R. Prestin-driven cochlear amplification is not limited by the outer hair cell membrane time constant. Neuron 70, 1143-1154. https://doi.org/10.1016/j.neuron.2011.04.024 (2011).

70. Dallos, P., Evans, B. N. \& Hallworth, R. Nature of the motor element in electrokinetic shape changes of cochlear outer hair cells. Nature 350, 155-157 (1991).

71. Huang, G. \& Santos-Sacchi, J. Mapping the distribution of the outer hair cell motility voltage sensor by electrical amputation. Biophys. J. 65, 2228-2236 (1993).

72. Santos-Sacchi, J. \& Song, L. Chloride and salicylate influence prestin-dependent specific membrane capacitance: Support for the area motor model. J. Biol. Chem. https://doi.org/10.1074/jbc.M114.549329 (2014).

73. Bai, J. P., Navaratnam, D. \& Santos-Sacchi, J. Prestin kinetics and corresponding frequency dependence augment during early development of the outer hair cell within the mouse organ of Corti. Sci. Rep. 9, 16460. https://doi.org/10.1038/s41598-019-52965-1 (2019).

\section{Acknowledgements}

This research was supported by NIH-NIDCD R01 DC016318 (JSS) and R01 DC008130 (JSS, DN). Thanks to

Kuni Iwasa and Rick Rabbitt for discussions. 


\section{Author contributions}

J.S.S. designed and performed experiments, analyzed data and wrote paper. D.N. edited paper. W.T. performed experiments, analyzed data and edited paper.

\section{Competing interests}

The authors declare no competing interests.

\section{Additional information}

Correspondence and requests for materials should be addressed to J.S.-S.

Reprints and permissions information is available at www.nature.com/reprints.

Publisher's note Springer Nature remains neutral with regard to jurisdictional claims in published maps and institutional affiliations.

(c) (i) Open Access This article is licensed under a Creative Commons Attribution 4.0 International License, which permits use, sharing, adaptation, distribution and reproduction in any medium or format, as long as you give appropriate credit to the original author(s) and the source, provide a link to the Creative Commons licence, and indicate if changes were made. The images or other third party material in this article are included in the article's Creative Commons licence, unless indicated otherwise in a credit line to the material. If material is not included in the article's Creative Commons licence and your intended use is not permitted by statutory regulation or exceeds the permitted use, you will need to obtain permission directly from the copyright holder. To view a copy of this licence, visit http://creativecommons.org/licenses/by/4.0/.

(C) The Author(s) 2021 